\title{
Diabetic Cardiomyopathy: A New Perspective of Mechanistic Approach
}

Yuvashree Muralidaran and Pragasam Viswanathan*

Renal Research Lab, Centre for Biomedical Research, School of Biosciences and Technology, VIT University, Vellore, Tamil Nadu, India

\begin{abstract}
Diabetic cardiomyopathy (DCM), one among the macrovascular complications of diabetes is the leading cause of mortality around the globe. The emergence of DCM irrespective of other cardiovascular diseases (CVD) necessitates a deeper insight into its occurrence and mechanism of development. This review summarizes the different stages in DCM progression based on molecular, cellular and functional level. The early stage in DCM involves oxidative stress and endoplasmic reticulum stress concomitant with O-linked glycosylation of receptors, and extracellular matrix (ECM) proteins, and formation of advanced glycation end products (AGE). This further influences myocyte apoptosis and steatosis, which is followed by fibrosis and remodeling, thus altering the physiological functioning of heart. It has been postulated that, microbuminuria (MA) which is an indicator of renal failure is also an early predictor of DCM. Currently, focus on epigenetic modifications, as observed in diabetics with renal failure is of critical importance to unveil the mystery behind the association of DCM and MA. Albeit, we examine DCM in a mechanistic approach, the major reason that remains unnoticed is glycemic excursion (GE). ACCORD, DCCT and EDIC studies have reported that the persistent effect in CVD is due to transient hyperglycemia. Hence it is predicted that, treatment strategies focusing on genetic level, along with maintaining persistent blood glucose would be more effectual. Besides, designing treatment regimen for patients with renal failure would be a preventive strategy for diabetic cardiomyopathy when their state towards DCM incidence is also considered and monitored precisely.
\end{abstract}

Keywords: Diabetic cardiomyopathy; Cardiovascular diseases; Microalbuminuria; Renal failure; Hyperglycemia

\section{Introduction}

Diabetes is the leading cause for pandemic rise in various micro- and macro-vascular complications, embodying social and economic burden worldwide. Diabetes related heart disease includes coronary heart disease, cardiac autonomic neuropathy or diabetic cardiomyopathy (DCM) [1]. Diabetic cardiomyopathy is one of the major causes for end stage heart failure, marked by left ventricular (LV) compliance and abnormal ejection fraction, leading to sudden cardiac arrest and death. Decades ago, Rubler et al. coined the term "diabetic cardiomyopathy" based on postmortem findings of four diabetic cases reported with ventricular hypertrophy and hyperfiltration [2]. This functional loss of left ventrical due to diabetes signifies myocardial damage that exists independent of other vascular diseases [3-6,7].

Amongst all other comorbidities of heart failure, mortality caused due to cardiomyopathy under diabetic condition is reportedly high, where DCM was evident in $60 \%$ of type 2 diabetes mellitus (T2DM) cases. It has been estimated that, worldwide, the prevalence of diabetes mellitus (DM) will increase from $2.8 \%$ in 2000 to $4.4 \%$ in $2030[8,9]$. It has been postulated that DCM is marked by asymptomatic diastolic dysfunction referred to as preclinical diastolic dysfunction (DD), followed by systolic damage in diabetics [10]. This diastolic dysfunction in diabetic patients is ascertained to be related to aging and duration of diabetes rather than hypertension and left ventricular hypertrophy [11].

An appreciation on understanding the structural abnormalities that arise in progression of myocardial dysfunction, and the molecular mechanism behind these, is vital for an effectual amelioration of DCM. Whilst, pathogenesis of cardiomyopathy is well studied by researchers, understanding on pathophysiology related to these morphological alterations is conceived to identify a better marker at an earlier stage [12]. This review focuses on how heart carries out certain adaptive measures to combat the impact of diabetes, whether these measures are really adaptive or maladaptive, and relates diabetes mediated microalbuminuria to cardiomyopathy. Further we discuss on epigenetic changes influencing the structural and functional abnormalities in diabetic cardiomyopathy.

\section{DCM Cardiomyopathy: Possible Stages}

Diabetic cardiomyopathy has been classified into stages (Figure 1), based on ejection fraction and phenotypic changes in left ventricles (LV). The initial stage of DCM is asymptomatic marked by LV hypertrophy with normal ejection fraction, followed by stage 2 with noticeable dilatation and reduced ejection fraction, patients with systolic and diastolic dysfunction mediated by contributing factors viz micro-angiopathy, hypertension and myocarditis are considered in stage 3 and the final stage designated as end-stage or refractory heart failure with ischemia, infraction and remodeling [12].

This stepwise progression of DCM has been engendered due to hyperglycemia mediated metabolic dysregulation, ensuing cardiac apoptosis, necrosis, fibrosis, steatosis, hypertrophy and remodeling [13]. Such structural modifications influence cardiac dysfunction characterized by an initial diastolic dysfunction followed by altered systolic function.

*Corresponding author: Dr. Pragasam Viswanathan, Professor, Renal Research Lab, Centre for Biomedical Research, School of Biosciences and Technology, VIT University, Vellore - 632 014, Tamil Nadu, India, Tel: +91-416-2202583; E-mail pragasam.v@vit.ac.in; pvishvanath@yahoo.com

Received August 30, 2015; Accepted September 10, 2015; Published September 15,2015

Citation: Muralidaran Y, Viswanathan P (2015) Diabetic Cardiomyopathy: A New Perspective of Mechanistic Approach. J Diabetes Metab 6: 605. doi:10.4172/2155 6156.1000605

Copyright: (c) 2015 Muralidaran Y, et al. This is an open-access article distributed under the terms of the Creative Commons Attribution License, which permits unrestricted use, distribution, and reproduction in any medium, provided the original author and source are credited. 


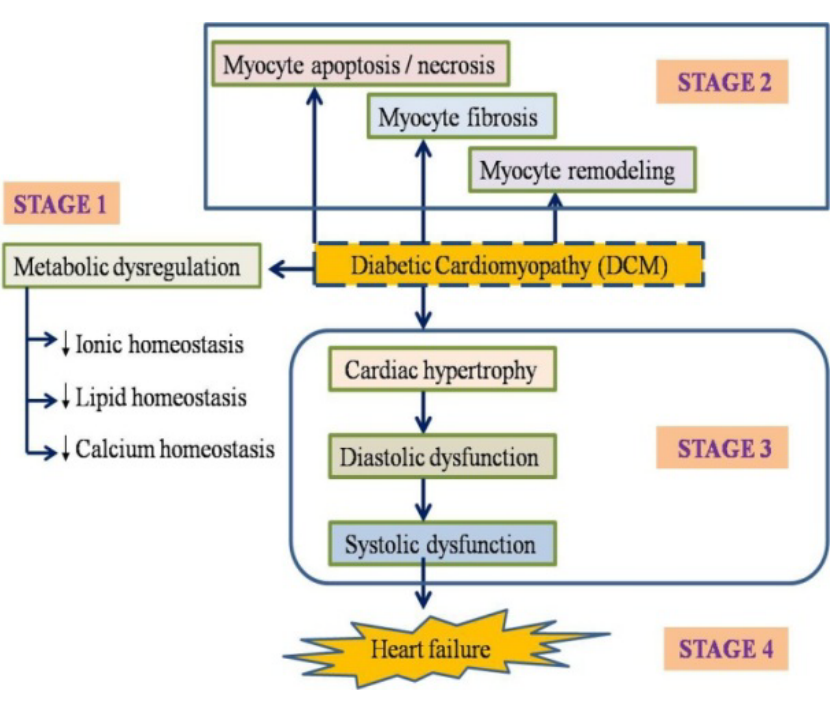

Figure 1: A summary of different stages in diabetic cardiomyopathy.

\section{Diastolic Dysfunction}

Diastolic dysfunction (DD) marked by ventricular stiffness and impaired relaxation is recognized as the earliest manifestation of DMinduced LV dysfunction. Diabetes mediated cardiac structural changes has been evidenced as a major cause of diastolic dysfunction, with its prevalence being $21 \%$ to $75 \%$ in diabetic cases $[13,14]$.

Diastole starts at closure of aortic valve, along with LV filling, atrial contraction and subsequent ventricular relaxation. Thus diastolic dysfunction culminates in elevated filling pressure with mean pulmonary capillary wedge pressure (PCWP) being $>12 \mathrm{~mm} \mathrm{Hg}$ or when the left ventricular end-diastolic pressure (LVEDP) being $>16 \mathrm{~mm}$ $\mathrm{Hg}$ [15]. In normal heart with normal load the ventricular filling nearly ends at minimal pressure. The elevated filling pressure is mainly due to elevated after load (pressure generated in left ventricles to overcome aortic pressure) along with elevated preload (end-diastolic pressure, stretching of ventricles prior to contraction) [16]. Myocardial stiffness is another factor influencing diastolic dysfunction that modulates the cardiac contraction. This is caused due to the increased deposition of advanced glycation end products and increased resting tension [17].

There are various methods to assess diastolic dysfunction, which includes tissue doppler imaging, pulse-wave doppler, cardiac magnetic resonance and colour M-mode propagation velocity. Diastolic dysfunction is classified into stages as follows: impaired relaxation, pseudonormal, reversible restrictive and fixed restrictive. The parameters to analyse these stages of DD include mitral inflow, pulmonary venous flow and valsalva maneuver. Of all, mitral E/A is widely used as marker for diastolic filling, which represents early to late filling velocities [18-20].

Using pulsed-wave Doppler across the mitral valve, the normal pattern of diastolic function (DD) measured is defined as E/A $>1$ in humans, which can also be observed at early stage of ischemia and hypertrophy, impaired early diastolic dysfunction is denoted as E/A < 1 , pseudonormal pattern or moderate DD group as $\mathrm{E} / \mathrm{A}>1$ that occurs to compensate the impaired relaxation by increasing the early filling and LV filling pressure (LVFP) to maintain the cardiac output. The advanced grade of DD with restrictive filling pattern and elevated filling pressure is defined as $\mathrm{E} / \mathrm{A}>2[20,21]$.
Since diastolic dysfunction being the initial stage in DCM, early diagnosis of DD may prevent the disease progression.

\section{Systolic Dysfunction}

The ventricular myocardium is characterized by circular, oblique and longitudinal myocardial fibers with global LV functioning viz radial, circumferential and longitudinal. This LV function depends on LV size, wall thickness and heart rhythm [22,23].

It has been reported that in streptozotocin induced diabetic Sprague Dawley rats, diastolic dysfunction was prevalent in all diabetic rats 2 to 3 months from STZ induction. The average time from induction of DM to the development of both systolic and diastolic dysfunction was 9.2 months. Reduced acceleration in LV in correspondence with elevated wall segments and a normal LVEF were related to left ventricular dysfunctioning ensuing impaired cardiac contractibility [24].

A prospective, multi-centered study, The SHORTening of midWall and longitudinAl left Ventricular fibers in diabEtes (SHORTWAVE) trial with 386 type $2 \mathrm{DM}$ cases and 120 normotensive healthy volunteers has shown that, in diabetics, systolic dysfunction is associated with reduced shortening of longitudinal fibers and shortening of circumferential LV fibers that is dependent on increased stress [25]. Moreover longitudinal contraction is found to occur in early stage of DCM, since it is influenced by contraction of sub-endocardial fibres that are more susceptible to fibrosis. Earlier it was assumed that longitudinal shortening is followed by increased radial function that emerges to combat the longitudinal function. In contrary, Ernande et al. has demonstrated using spectral tracking imaging that in a prospective study of 112 type $2 \mathrm{DM}$ patients both radial function and longitudinal function occurs simultaneously, along with circumferential shortening [26].

Besides LV dysfunction, right ventricular (RV) dysfunction has also been observed in type 2 diabetic patients. Albeit, the reason behind $\mathrm{RV}$ dysfunction remains elusive, the abnormalities were found to be similar to LV dysfunction. Hence it has been conceived that fibrotic lesion may diffuse from LV to RV via sub-endocardial fibres present in the arterioventricular septum [27]. These ventricular remodeling initially arises to overcome the effects of interstitial fibrosis and to maintain a normal functioning of heart, which later on accounted for an elevated ventricular contractile dysfunction along with widely spread fibrotic lesions.

\section{Metabolic Dysregulation: Sign of Hyperglycemia}

The diabetic patients at high risk of developing heart failure are characterized with altered metabolism in cardiomyocytes (Figure 2). Albeit, heart energetics depend on various substrates depending on the dietary and physiological conditions, fatty acid and glucose are crucial among them, wherein utilization of either has a reciprocal inhibitory effect on another termed as "Randle cycle". Normal adult heart generates $60 \%-80 \%$ ATP by fatty acid $\beta$-oxidation and remaining from glucose oxidation. The uptake of free fatty acids (FFA) is via 2 modes, simple diffusion or FAT/CD36 transporters which accounts for $80 \%$ of FFAs uptake, of which $75 \%$ of FFA gets oxidized immediately and is esterified to fatty acyl CoA by various fatty acyl CoA synthase (FACS) enzymes, which is then channeled to mitochondria via 2 enzymes, outer membrane bound carnitine palmitoyl transferase 1 (CPT1) that converts it into respective carnitine acyl CoA moieties, and carnitine translocase. The fatty acid $\beta$-oxidation generates NADH and acetyl CoA that are diverted to krebs cycle, involved in energy production [28]. 


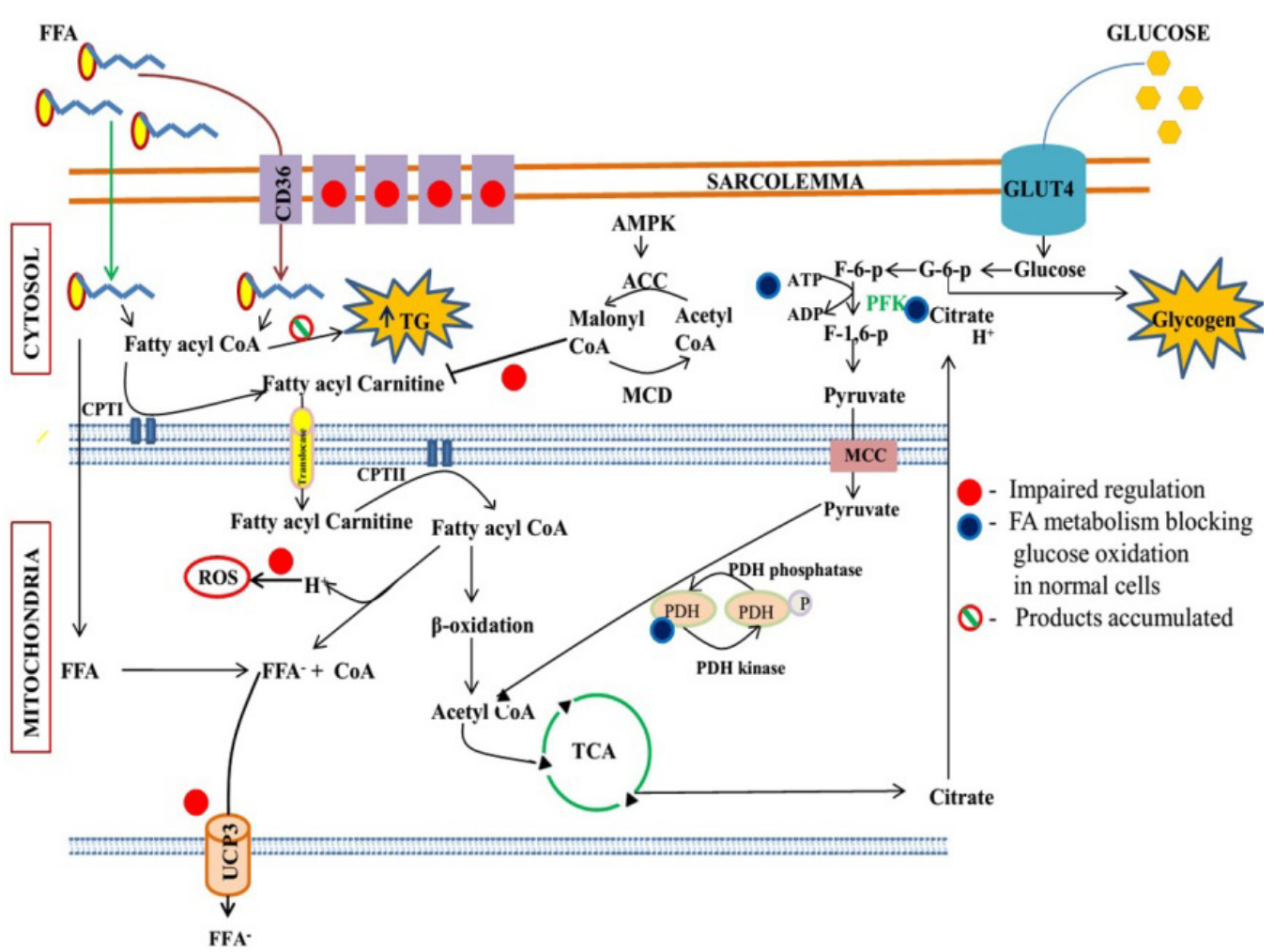

Figure 2: Schematic representation of substrate metabolism in myocytes. Hyperglycemic milieu causes elevated CD36 recruitment on the membrane, increasing fattyacid (FFA) uptake. Impaired regulation due to increased FFA uptake involves increased malonyl-CoA, inhibiting FA oxidation, and increased ROS formation. This further influences triglyceride (TG) accumulation and glycogen formation due to decreased glucose oxidation. Abbreviations: MCD - Malonyl CoA decarboxylase, ACC - Acetyl-CoA, CPT1 - Carnitine palmitoyl transferase 1, MCT - Mono-carboxylase carrier.

Although FFA is the predominant energy source in heart, glucose utilization also plays a significant role in maintaining the cardiac plasticity and energy homeostasis. Glucose contributes to energy production via glycolysis, pyruvate the end product of glycolysis is converted to either acetyl-CoA or lactate depending on aerobic or anaerobic condition respectively. Further, glycolysis is regulated by activation of pyruvate dehydrogenase that is inhibited by pyruvate dehydrogenase kinase (PDH kinase) and fatty acid oxidation. Indeed, fatty acid $\beta$-oxidation is endogenously regulated by malonyl$\mathrm{CoA}$, which is being synthesized from acetyl-CoA (generated from peroxisomes) by acetyl-CoA carboxylase (ACC) in the cytosol. This entire mechanism of regulation is mediated by AMPK. Thus AMPK is not only implicated in regulating cell survival mechanism, but also in energy substrate metabolism. Disruption in these regulated pathways is one of the reasons for myocardial dysfunction [28-30].

Further FAT/CD36, localized in the intracellular endosomal compartment straddles on the sarcolemma that facilitates fatty acid uptake. The effect of insulin and cardiac contraction has been found to be additive in regulating FAT/CD36 recruitment. Unlike contraction mediated CD36 recruitment, insulin mediated recruitment is found to be PI-3 kinase dependent. Further insulin mediated FAT/CD36 transmembrane expression has augmented FFA esterification to FFA oxidation ratio by increasing malonyl-CoA, the inhibitor of mitochondrial FFA uptake. This elevated FA esterification leads to increased triglycerides storage reserve, leading to insulin resistance $[31,32]$.

Insulin resistance, in general, decreases the capacity of myocytes to utilize glucose, thus reducing its substrate flexibility. Under diabetic condition, the inability of myoctes to take up glucose is compensated by its dependence on alternate source of energy for maintaining the functionality of heart. This is consistent with the finding that, under diseased condition membrane bound CD36 expression is increased, facilitating elevated fatty acid (FA) uptake, this condition is attenuated in CD36 knock-out MHC- PPAR- $\alpha$ mice that mitigated the lipotoxicity in cardiomyocytes by decreasing FA accumulation and deactivating subset of PPAR- $\alpha$ target genes [33].

It has been reported that though various FA-activated nuclear receptors PPAR- $\alpha$, PPAR $-\beta / \delta$ and PPAR- $\gamma$ are being expressed in heart, PPAR- $\alpha$ plays a pivotal role in FA accumulation under disease condition [34]. These nuclear receptor family receptors heterodimerizes on binding to fatty-acyl-fattyacyl-CoA, which upregulates fatty acid metabolism and as a compensatory mechanism it induces $\mathrm{I} \kappa \mathrm{B}$ expression that represses $\mathrm{NF} \kappa \mathrm{B}$ (proxidant gene) expression. PPAR- $\alpha$ agonist WY14643 has been substantiated to suppress inflammatory cytokines viz TNF- $\alpha$, MCP1 and macrophage antigen- 1 and augment adiponectin levels in myocarditis [35].

Uncoupling proteins (UCP), in particular UCP2 and UCP3, which are predominant in heart has been implicated in reducing mitochondrial membrane potential $(\Delta \psi)$ that influence ROS generation, besides it also protects the heart from exogenous oxidant stress [36]. It has been found that UCP wields ROS generation vividly by regulating fatty acid oxidation. Under elevated fatty acid oxidation, intra-mitochondrial fatty acyl CoA is cleaved by mitochondrial thioesterase to release CoA and FFA on release of a proton that decreases free radical generation. 
Attenuation of these profound metabolic derangements has shown to arrest its untoward effects viz structural and functional abnormalities [37]. Indeed, however, the role of UCP3 in diabetics remains elusive and has to be well studied.

\section{ER stress}

In another perspective, Insulin resistance in heart has been stated as an adaptive mechanism to overcome proteotoxicity and endoplasmic reticulum stress. As discussed by Taegtmeyer and Lubrano, dysregulated substrate metabolism causes metabolic stress that influences the protein turnover. In non-ischemic heart disease viz diabetes, there is an observed increase in glucose uptake to glucose oxidation, ensuing glucose-6-phosphate (G6P) accumulation and increased signaling through mTOR pathway causing elevated unfolded and misfolded proteins, subsequently leading to ER stress. Hence, it has been hypothesized that to overcome such effect, cardiac muscles undergo an adaptive mechanism of insulin resistance [37].

ER stress foster the activation of transmembrane ER resident proteins PERK, IRE-1 and ATF6 by increasing the dissociation of ER localized molecular chaperon GRP78 that is attached to the N-terminal domain of these ER resident protein on the luminal side, and by increasing the affinity of GRP78 towards the unfolded proteins. This unfolded protein response (UPR) is vital to overcome the deleterious effects of unfolded or misfolded proteins. However, the outcome of UPR depends on its phase of action either prosurvival phase or proapoptotic phase. The apoptosis of myocytes under ER stress is energetised via the activation of JNK pathway, caspases- 12 and by suppressing the activity of proapoptotic proteins [38]. GRP 78 and caspases-12 has been found to be aggravated in diabetic cardiomyopathy that evidences the activation of ER stress mediated apoptosis. Besides, the decreased myocyte regeneration capacity in adult cardiac muscles and loss of endothelial cells exacerbates cardiac dysfunction [39].

Whilst, insulin resistance is enunciated to be an adaptive process under hyperglycemia mediated ER stress, it seems to be counterintuitive, since ER stress is also invoked by the underlying effects of hyperglycemia. Indeed, the effect of insulin resistance is even more adverse, this culminates in elevated free radical generation, eventually leading to oxidative stress. Further, insulin resistance engenders glucose uptake via insulin insensitive transporters, that channels glucose via alternate pathways of glycolysis.

\section{Oxidative stress}

Oxidative stress remains a causative factor for complications of diabetes. The driving force for oxidative stress in hyperglycemic conditions is the reductive stress marked by NADH accumulation due to impairment of complex 1 in electron transport chain $[40,41]$. This leads to a condition called pseudohypoxia, where the cells does not respond properly to the presence of oxygen. The interrelationship between hypoxia and hyperglycemia makes the condition worse, compared to their individual effects, because of the increase in lactate production under hypoxic condition [42]. Further Zhang et al., has demonstrated that fatty liver is more prone to damage due to the sorbitol accumulation under hypoxia [42].

The insulin independent cells take up the high glucose and channel them via branch off pathways of glycolysis like polyol, hexosamine and methylglyoxal pathway. This eventually increases the rate of superoxide radical ( $\mathrm{SO}$ ) formation or glucose gets stored as glycogen reserves reducing glucose oxidation [43].

Branch off pathways of glycolysis: The cause for impaired glucose transport under high glucose ambience depends on the differential tissue specific expression of GLUT transporters. About $28 \%$ of basal GLUT4 is membrane associated in healthy heart, majority of GLUT transporters in cell surface of myocardium consists of GLUT12 in healthy and diabetic cases that functions similar to GLUT1 transporters, which are insulin insensitive. This has been demonstrated by progressive bio-tinylated photolabeling, where in insulin stimulation did not have any effect on GLUT12 translocation in perfused heart or in isolated myocytes [44], in contrary cell surface GLUT12 expression is found to be increased in diabetic cases. This altered glucose transport via

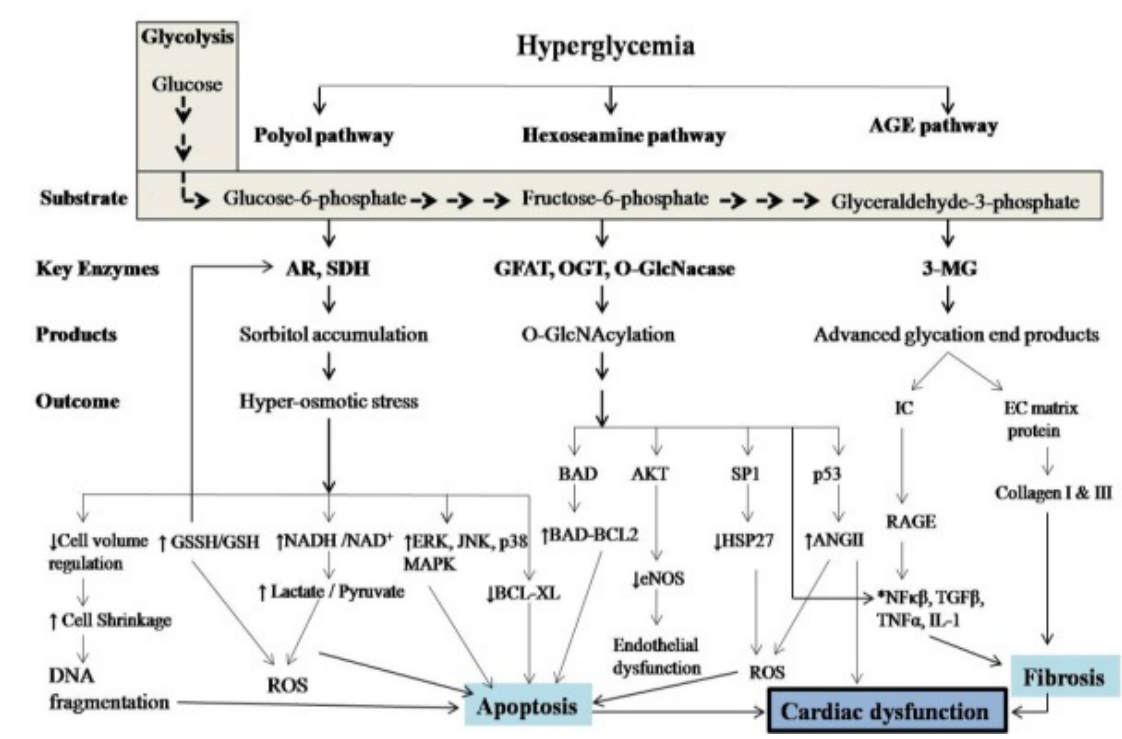

Figure 3: Schematics of branch off pathways of glucose metabolism under hyperglycemic milieu and sequential events influencing myocyte apoptosis and fibrosis resulting in cardiac dysfunctioning. Enzymes involved in the metabolism include - Aldose reductase (AR), Sorbitol dehydrogenase (SDH), Glutamine:fructose-6phosphate aminotranferase (GFAT), O-GIcNAc transferase (OGT), N-acetylglucosaminidase (O-GIcNAcase). ${ }^{*}$ indicates activation by HMP and AGE products formed. 
GLUT1 and GLUT12 transporters indicate increased glucose uptake and insulin resistance that represents metabolic abnormality $[45,46]$.

The glucose transported via such basal transporters (not stimulated by insulin) gets to be channeled across the branch off pathways of glycolysis (Figure 3). About $30 \%$ of glucose is consumed via polyol pathway, where in sorbitol dehydrogenase utilizes $\mathrm{NAD}^{+}$for sorbitol oxidation. This makes $\mathrm{NAD}^{+}$unavailable for glyceraldehyde-3phosphate dehydrogenase (GADPH) activity. The subsequent NADH formed is then taken up by NADH oxidases that lead to ROS generation causing further impairment in GAPDH by modifying the cysteine residue, thus leading to accumulation of glyceraldehydes-3-phosphate (G3P) in glycolysis [47].

The NADPH utilizing catalytic activity of aldose reductase (AR), a rate limiting step in polyol flux, is another major source for ROS generation, which reduces the formation of oxidized glutathione, a primary ROS scavenger. Tiwari et al., has reported that high glucose induced sorbitol accumulation influenced the hemolysis of human erythrocytes and altered the redox ratio in retinopathy induced goat lens; this effect is extenuated by the antioxidant property of certain vegetable juices [48]. Besides, sorbitol accumulation has been found to induce hyperosmotic stress in cardiomyocytes that culminates in DNA fragmentation and cell shrinkage. These changes were mediated by upregulation of P13K/PKB and MAPKs: Extracellular signal-regulated kinase (ERK), P38-MAPK and c-Jun N-terminal kinase (JNK) isoforms (P46, P54), and by downregulation of anti-apoptotic protein BCL-2 [49].

Hexosamine biosynthesis pathway (HBP) is postulated to be a nutrient sensor that regulates the flux of glucose, FFAs, uridine and glutamine into the cell. Unlike other accessory pathways of glycolysis, HBP leads to glycosylation of cytoplasmic and nuclear proteins [50]. This glycosylation process is regulated by two key enzymes $O-G l c N A c$ transferase (OGT) and $\mathrm{N}$-acetylglucosaminidase (O-GlcNAcase) [51,52].

About $2-5 \%$ of glucose conducted via this pathway is associated with O-GlcNAcylation of transcriptional factors, cytokines and insulin signaling molecules that plays a key role in diabetic complications. These in turn upregulates transcriptional factors ilk TGFa, TGF $\beta$, leptin, PAI-1 and decreases phosphorylation of Akt and GSK3. Enhanced HMP flux modified Specificity factor (SP1) with O-linked glucosamine (O-GlcNAc) that binds to various promoters, exacerbating hyperglycemic condition. Such promoter region includes adipocytokine and heat shock protein (HSP). SP1 has found to reduce HSP27 expression elevating reactive oxygen species (ROS) generation leading to apoptosis. Further in diabetes, SP1 expression has found to be enhanced that is positively correlated with the development of insulin resistance [50,53]. Zimmerman and Harris, has demonstrated that high glucose induced HBP flux negatively regulates leptin dependent phosphorylation of STAT3 (S727), implicating leptin resistance [54].

In contrary, O-GlcNAcylation is found to be cardioprotective in acute elevation, and the adverse effect shows up in chronic condition or increased frequency of occurrence. Majumdar et al. has reported that SP1 glycosylation and its translocation into nucleus transactivated (calmodulin) CAM gene in liver cells, which is mediated by insulin [55]. So, the impact of elevated HBP flux mediated glycosylation causing insulin resistance would also suppress the calmodulin expression. Further insulin resistance has been demonstrated to induce mitochondrial dysfunction that failed to oxidize glucose and fatty acid for energy [56].
It has been found that O-GlcNAcylation and phosphorylation compete for the same site in protein, and the response elicited due to enhanced glycosylation under hyperglycemic milieu is found to be comparatively robust. Consistent with these findings, Zeiden et al. has reported that, augmented O-GlcNAcylation of IKappa $\beta$ kinase complex $(\mathrm{IKK} \beta)$ has been observed in diabetics with elevated NFK $\beta$ expression [57]. Besides, O-GlcNAcylation of pro-apoptotic protein BAD has found to be involved in BAD-BCL2 dimerisation by inhibiting BAD phosphorylation, thus suppressing BCL2 activity causing apoptosis. Further, nuclear protein P53 (tumor suppressor protein) glycosylation has been observed concomitant with elevated angiotensin II (ANGII) synthesis that leads to myocardial apoptosis [58]. Contrarily, Marsh et al. has reported that augmented O-GlcNAcylation has led to decreased ANGII synthesis that is implicated in impaired hypertropic signaling, and is found to be independent of P53 expression [59].

Indeed, hyperglycemia also induces functional and structural change in microvasculature by mediating O-linked glycosylation of nitric oxide synthase that decreases AKT dependent phosphorylation of endothelial nitric oxide synthase (e-NOS) and histamine induced NO mediated dilation of arterioles. In addition to O-GlcNAcylation of proteins in the vasculature, HBP shunt is further associated with altered myocardial substrate utilization. Glucosamine increased O-GlcNAcylation and transport of FAT/CD36 (fatty acid transporters) on cell surface that is related to increased fatty acid uptake with concomitant decrease in carbohydrate uptake as discussed earlier [60]. So it can be concluded that though insulin recruits CD36, it might not be its only activator, and insulin resistance decreased only GLUT4 mediated glucose uptake, but not CD36 mediated FA uptake. Further, cardiac related proteins troponin I and myosin-actin heavy chain has been found to be glycosylated in diabetic condition. In addition to glycosylation, uridine diphosphate- $\beta-N$-acetylglucosamine (UDPGlcNAc) formed in HMP flux also mediates synthesis of proteoglycan and glycoprotein in endoplasmic reticulum and golgicomplex [60].

Many studies have confirmed the deleterious effect of advanced glycation end products implicated in disease progression. These AGEs are generated intracellularly and extracellularly by non-enzymatic glycation of protein molecules termed "Maillard reaction", in which sugar such as glucose reacts with protein amino group to form schiff base. The Schiff base then converts to a more stable Amadori product and the subsequent rearrangement of Amadori products leads to the formation of stable and irreversible AGE compounds. These AGEs formed covalently attaches to extracellular matrix proteins, especially collagen leading to myocardial stiffness that precedes myocardial fibrosis. Elevated collagen III to collagen I has been reported in diabetic heart. The extracellular AGEs formed binds to RAGE and elicits cascade of reactions by ROS generation that mediates NF- $\kappa \beta$ induced inflammation and apoptosis, along with synthesis of inflammatory cytokines IL- 1 and TNF- $\alpha$ and profibrotic cytokines TGF- $\beta$, inducing extracellular matrix protein synthesis. Further intracellular AGE formed affects the $\mathrm{Ca}^{2+}$ ATPase affecting the contractibility of myocardium [61].

The crosslink breaker of preformed AGEs, 3-phenacyl-4,5dimethylthiazolium chloride (ALT-711), has been manifested to attenuate the receptors RAGE and AGE-R3 expression and reduce CTGF expression in diabetic hearts. Additionally, aminoguanidine and ALT-946, the anti-glycation drugs against diabetic nephropathy have been shown to ameliorate the myocardial stiffness by reducing collagen-AGE formation, thus mitigating ventricular dysfunction [61].

Thus hyperglycemia has been found to induce cadiomyopathy by 
elevated oxidative stress, by enhancing ROS generation and decreasing antioxidant enzymes. Azelnidipine (AZL), an antioxidant has attenuated these changes by increasing Mn-SOD and Bcl-2 expression, and by decreasing p67phox, NADPH oxidase subunit recruitment to the membrane [62]. This shows that diabetes induced oxidative stress involves both NADPH oxidases and mitochondria that is further linked to apoptosis.

\section{Autophagy and Apoptosis}

Recent studies have enlightened the cause of apoptosis in myocytes under high glucose ambience. Generally, autophagy is considered as a catabolic process that is activated under cellular stress like glucose deprivation and pathogenic infection. Current demand on understanding the role of autophagy in high glucose mediated metabolic disorders is growing eminent due to its role in cell adaptation, cell survival and cell death $[63,64]$.

Generally, in elongating autophagosome membrane inactive unconjugated form LC3B is transformed into the phosphatidylethanolamine-conjugated form (LC3BII). More than 30 Atg (autophagy-related gene) gene regulated proteins have been substantiated in inducing autophagosome formation and maturation [65]. The ratio of LC3BII to LC3BI measures the autophagy packaging activity. Further, p62, which is an adapter molecule tethers proteins and organelles to autophagosome via binding to LC3B. Albeit, autophagy observed in diabetic rats in earlier stages occurs to be a protective mechanism that functions by phagocytosing the misfolded proteins by polyubiquitination, its role in later stages remains elusive. A concomitant increase in autophagosome with progression of cardiomyopathy was analyzed by measuring the membrane associated microtubule-associated protein-1 light chain-3 (LC3), a fluorescent autophagosome marker, to cytosolic LC3. However, elevated autophagy under high glucose is positively correlated with caspase-3, a marker for apoptosis [66]. This indicates augmented autophagy is related to progression of apoptosis.

Contrarily, Xie et al. has demonstrated that the progression of cardiomyopathy is related to decreased autophagy, and this condition is ameliorated by administering metformin [67]. This is consistent with the evidence that, under high glucose milieu, upregulated hexose biosynthesis pathway increased OGlcNAcylation, this blunted the beclin-1 expression. BCL-2 (Bcell lymphoma 2), Beclin1 and BNIP3 have been known to be key mediators of apoptosis and autophagy. OGlcNAc of these proteins further affects the hypertrophic signaling pathways. Glucose deprivation in diabetic cardiomyocytes blunted Beclin1 and LC3-II response indicating impaired autophagy, which shows the abnormal response of heart under diabetic condition [68]. This suggests that in hyperglycemic condition, the increase in autophagy as determined earlier, would be due to accumulated autophagosomes with impaired function. Another prominent regulator of autophagy is AMPK (AMP activated protein kinase) that decreases mTOR1 mammalian target of rapamycin kinase activation which augments autophagy by phosphorylating unc51-like kinase $1 / 2$ (ULK1/2, the mammalian homolog of yeast autophagy-related gene 1 [Atg1]). This results in translocation of ULK1/2 to ER (endoplasmic reticulum) membrane, which further recruits E3 like Ubiquitin ligase along with E2 and E1 that facilitates LC3-II formation, involved in autophagosome elongation. The pivotal step in initiating autophagy is the fusion of lysosome and autophagosome forming autolysosome. Along with several proteins, Rab7 and Vsp34-Beclin1 complex has been found to activate autophagosome maturation. In diabetic heart, impaired autophagy due to accumulation of autophagosome has been found to be due to impaired expression of these proteins [69,70]. Consistent with this finding, hyperglycemia has been manifested to decrease AMPK in H9c2 cadiomyocytes, inhibiting JNK-BCL2 signaling, thus inducing apoptosis and suppressing autophagy by promoting Beclin1BCL2 interaction. Metformin is found to normalize AMPK expression

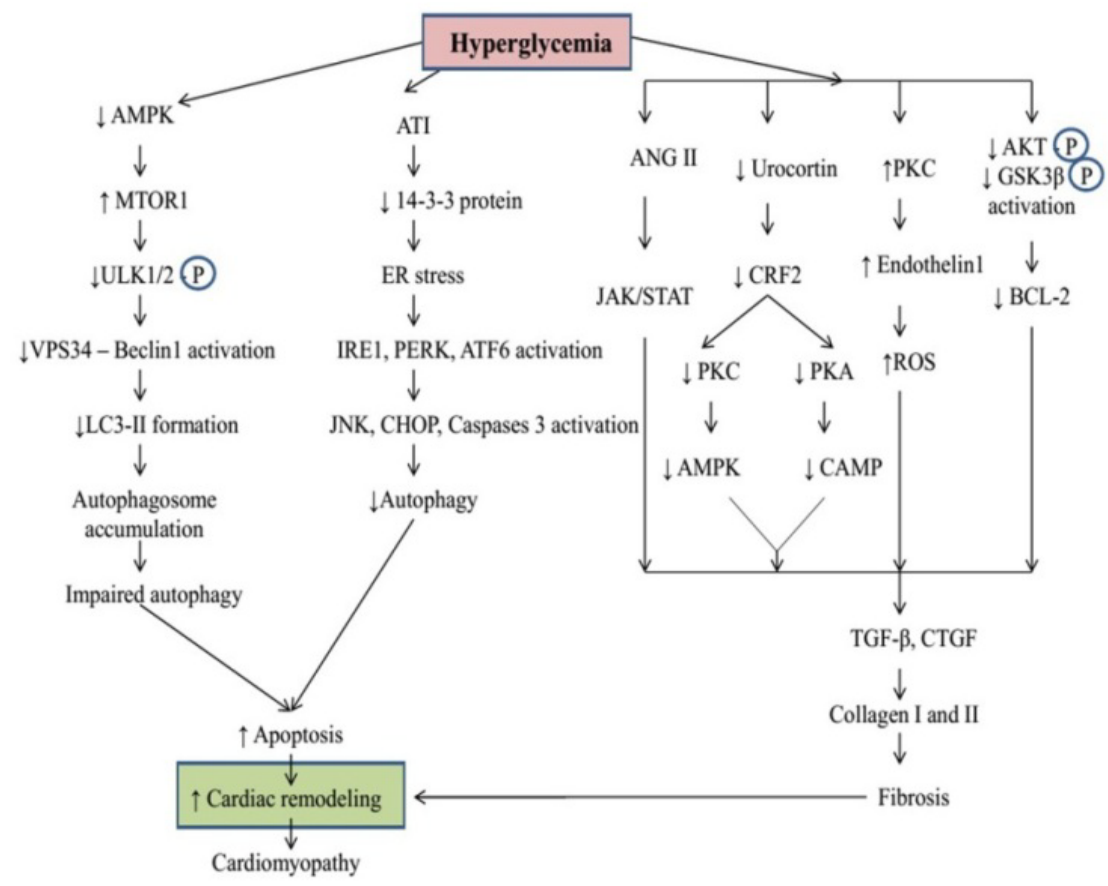

Figure 4: Summary of events involved in progression of diabetic cardiomyopathy. Schematic depicting the altered expression of signaling proteins that mediate impaired autophagy, increased apoptosis and fibrosis leading to cardiac remodeling, that modifies the physiological functioning of heart. 
and promote $\mathrm{BCL} 2$ phosphorylation, which is required to maintain the integrity of mitochondrial outer membrane and prevents the release of proapoptotic factors salvaging cells from apoptosis (Figure 4) [71,72].

Such phagosomal dysfunction analysed in diabetic heart is marked by increased p62 expression and decreased SIRT1 (sirtuin 1) expression. Reservetaol has been found to increase SIRT1 expression mediated deacetylation and HDAC mediated deacetylation of FOXO1, facilitating FOXO1 transcriptional activity and stimulating autophagic flux. FOXO1 in turn regulates Rab7 expression, which acts as a major factor in autophagosome maturation and phagolysosome formation [73]. Besides, SIRT1 has been substantiated to deacetylate peroxisome proliferator-activated receptor-gamma coactivator 1 alpha (PGC$1 \alpha)$ and induce the downstream target genes, such as the peroxisome proliferator-activated receptor gamma (PPAR $\gamma$ ) gene that is involved in uptake of fatty acids and in energy homeostasis [74]. Hence decreased phagosomal functioning should be concomitant with impaired energy metabolism, and it is found that nutritional stress contributes to enhanced autophagy that ravens the harmful metabolites and wornout organelle [75].

The impaired metabolism in myocytes is marked by buildup of glycogen reserve, enhancing autophagy of glycogen, termed as glycophagy. Insulin stimulates glycogen synthesis via AKT mediated inhibition of glycogen synthase kinase $3 \beta$. However, under insulin resistance, high glucose mediated glycogen storage is found to be independent of AKT and is concomitant with augmented starchbinding domain containing protein-1 expression, marker for glycophagy involved in binding glycogen and mediating membrane anchorage via interaction with the cognate protein $\mathrm{GABA}(\mathrm{A})$ receptor-associated protein-like 1 (GABARAPL1). AMPK as it regulates autophagy has also found to regulate glycogen storage by augmented glucose uptake and activating glycogen synthase. However, autophagosome formation is found to be independent of glycophagy. Besides, the suppressed AMPK activity in DCM should be converged with decreased autophagy and glycogen storage, but the counterintuitive mechanism of elevated glycogen storage linked glycophagy suggests that, it should be independent of AMPK or AKT activation. Subsequently, decreased glucose oxidation has been found to substantiate elevated glycophagy [76,77].

Heme oxygenase (HO-1) is among these enzymes that increase in patients with diabetes. HO-1, the rate-limiting enzyme in heme degradation, catalyses the oxidation of heme to generate several biologically active molecules, such as carbon monoxide (CO), biliverdin, and ferrous ion. Evidences have shown that, HO1 has been implicated in autophagy that is regulated by AKT mediated AMPK activation, potentiating autophagy [78].

TNFSF10 (tumor necrosis factor-related apoptosis-inducing ligand) has been known to induce apoptosis, is now found to be a regulator of autophagy. In cancer cells TNFSF10 inhibits apoptosis and promotes autophagy via activation of anti-apoptotic proteins and by blocking apoptotic signaling. Further autophagy is regulated via TRAF2 (TNF receptor associated factor 2) and RIPK2 (Receptorinteracting serine/threonine-protein kinase 2) activation, mediated by TNFSF10 induced MAPK8 activation. Knockdown of these proteins has potentiated TNFSF10 induced cytotoxicity by enhancing DISC (Death-inducing signaling complex) formation. As the autophagic flux is considered vital in controlling the disease progression, more insights into the mechanism of autophagy activation and designing drugs targeting the pathways is conceived to root for an effective treatment [79].
Autophagosome also targets particular organelles and phagocytose it, when mitochondria are being engulfed it is termed as "mitophagy". In diabetic cardiomyopathy, primarily, mitochondria is most prone to damage due to ROS generation, and this damaged or injured mitochondria has to be removed to curb the overproduction of ROS level. These abnormalities are followed by dedifferentiation and regeneration of the myofibrillar apparatus. The dominant source of cardiomyocyte turnover is reported to be from preexisting cardiomyocyte but not the progenitor cells. The increased rate of turnover during injury consequently leads to the utter functional loss in myocardium in diabetic milieu. Along with this fact, the myocyte mitosis and cytokinesis of pre-existing abnormal myocytes during regeneration has been found to worsen this condition [80].

\section{Fibrosis}

Eventually these abnormalities cause interstitial fibrosis, an irreversible morphological change, which exacerbates the cardiac dysfunction. Necrosis and apoptosis of myocytes has been elucidated as the cause for fibrosis formation. Under hyperglycemic ambience, the increased angiotenin II has been found to trigger JAK/STAT pathway, mediating augmented TGF- $\beta$ expression, which is the instigator of fibrosis that promotes collagen I and collagen II synthesis (Figure 4).

In addition to TGF- $\beta$, CTGF has also been elevated in concomitance with creatine phosphokinase isozyme (CK-MB), plasma brain natriuretic peptide (BNP), myocardial collagen volume fraction $(\mathrm{CVF})$ and left ventricular mass index (LVWI), indicating myocardial remodeling. These changes have been positively correlated with reduced phophorylation of AKT and GSK-3 $\beta$ that promote transcription of BCL-2, an antiapoptotic protein. Administration of urocortin or losartan (AT1 receptor antagonist) has been found to alleviate this condition [81]. Further augmented HMP shunt has been implicated in elevated glycogen glycosylation that is observed to increase fibrosis in cardiomyocytes, leading to cardiac stiffness promoting diastolic dysfunction.

Diabetes has also been conceived as a modulator of urocortin, an endogenous peptide, associated with corticotrophin releasing factor (CRF) family that activates CRF2 receptors, which is considered to be a potent vasodilator involved in cardiovascular functioning. Previous findings have reported that urocortin improves cardiac performance under ischemia/hypoxia. Hence its decrease in hyperglycemic condition would suggest that, high glucose should also cause hypoxia in cardiomyocytes, which might be due to blockage of coronary artery. Since, even a picomolar concentration of urocortin is anticipated to exert its effect due to its high affinity towards CRF2 receptor, it can be hypothesized that it is completely deteriorated in DCM. Thus treatment strategies targeting urocortin would prevent diabetes mediated cardiomyopathy and vascular dysfunction. In heart, Urocortin2 (Ucn2) has been substantiated to act via dual mechanism involving PKC mediated AMPK activation, followed by downstream AKT phosphorylation (Ucn-CRF2-PKC-AMPK-AKT) that is responsible for vasorelaxation, and via PKA mediated cyclic AMP production (Ucn-CRF2-PKA-CAMP), which would exert an inotropic effect [8183].

Likewise a potent vasoconstrictor endothelin1 (ET1), produced mainly from endothelial cells has been elevated under chronic hyperglycemia through PKC activation, which is found to be responsible for superoxide formation, and subsequent mitochondrial dysfunction and myofibrillar disarray in diabetic heart. Further, ET1 has been assessed to promote fibrosis by augmenting TGF- $\beta$ and CTGF 
expression, which mediates epithelial-mesenchymal transition and fibroblast accumulation. These fibroblasts have mainly considered as ET1 and collagen producing cells that has acquired a faster migrating capacity [84]. TGF- $\beta$ has been regarded as profibrotic gene that acts via smad2 phosphorylation, which is involved in de novo synthesis of collagen, plasminogen activator inhibitor 1 and biglycan, and in elongation of glycose-amino-glycan (GAG) chains in proteoglycan [85].

Further the functional changes in cardiomyocyte with respect to surrounding fibroblasts have been studied under impedence microscopy by Rother et al. [85]. The contractibility of cardiomyocytes is determined by analyzing the beating frequency, indicated as coupling. The increase in fibroblast is found to be coupled to decrease in contractile frequency. This is due to elevated spreading rate of fibroblasts concomitant with increased N-Cadherin and Cx43 (connexins) between them [86]. Thus augmented fibroblast production due to hyperglycemia may be the stage where functional loss of cardiomyocyte would emerge.

\section{Cardiac Contractibility and Remodeling}

Decades ago, the term Cardiac mechanical efficiency was expressed as work $/ \mathrm{MVO}_{2}$ that relates cardiac work to the energy consumed $\left(\mathrm{MVO}_{2}\right)$ by the ventricle during contraction [87]. This decreased mechanical efficiency indicates the impaired contractibility of cardiac muscles. Aforementioned, Polyol pathway not only attenuated the redox ratio but also the contractibility of cardiac tissue by decreasing the calcium reuptake by sarcoplasmic reticulum (SR) by inducing nitrosylation of tyrosine residue in SERCA (sarcoplasmic reticulum $\mathrm{Ca}^{2+}$ ATPase) pump and decreasing the s-glutathiolation, thus causing oxidation of cysteine-674 residue in SERCA [88].

As illustrated in (Figure 5), the accumulation of calcium is associated with the depletion of high energy phosphate stores and derangement of cellular ultrastructure that can lead to cardiac dysfunction [88]. SP1 also regulates the expression of specific isoform of SERCA in cardiomyocytes, which is observed to decrease during fetal development and increase under disease condition [89]. The localization of connexins in ventricles and $\mathrm{N}$-cadherins are mainly involved in signal transduction during cardiac contraction. These proteins involved in cell-cell contact, polarizes depending on mechanical situations, and its expression is also found to increase under stretch, but the cell orientation and elongation merely depends on such polarization. Whilst, $\mathrm{Cx} 43$ increased upon elevated angiotensin II and stretch in diseased, it resulted in a shift in polarization from cell poles to lateral sides, affecting the contractibility of myocytes. Thus only certain directed stretch is found to be responsible for proper polarization of Cx43. Cx43 are mostly localized in cell poles that enables proper signal transduction among cells. A shift in this site of polarization would culminate in functional abnormalities. So the direction of stress and polarization of connexin are pivotal in determining the contractibility of myocytes [90].

These structural changes causing asymmetric reorganization influence the cardiac functional abnormalities affecting the electrical conductivity that designates the impaired calcium handling and decreased calcium transients in myocytes. Recent study has shown that at an optimal stimulation frequency of $0.5 \mathrm{~Hz}$, which mimics diabetic condition, a decrease in myocyte shortening was observed, which might be due to decreased calcium transients observed after 4 to 12 weeks [91].

Further, contraction of muscle has been found to be responsible for glucose transport that is independent of SR $\mathrm{Ca}^{2+}$ release. The complete contraction is determined to be facilitated by increased AMPK and mechanical stress, which then combinedly and independently acts to

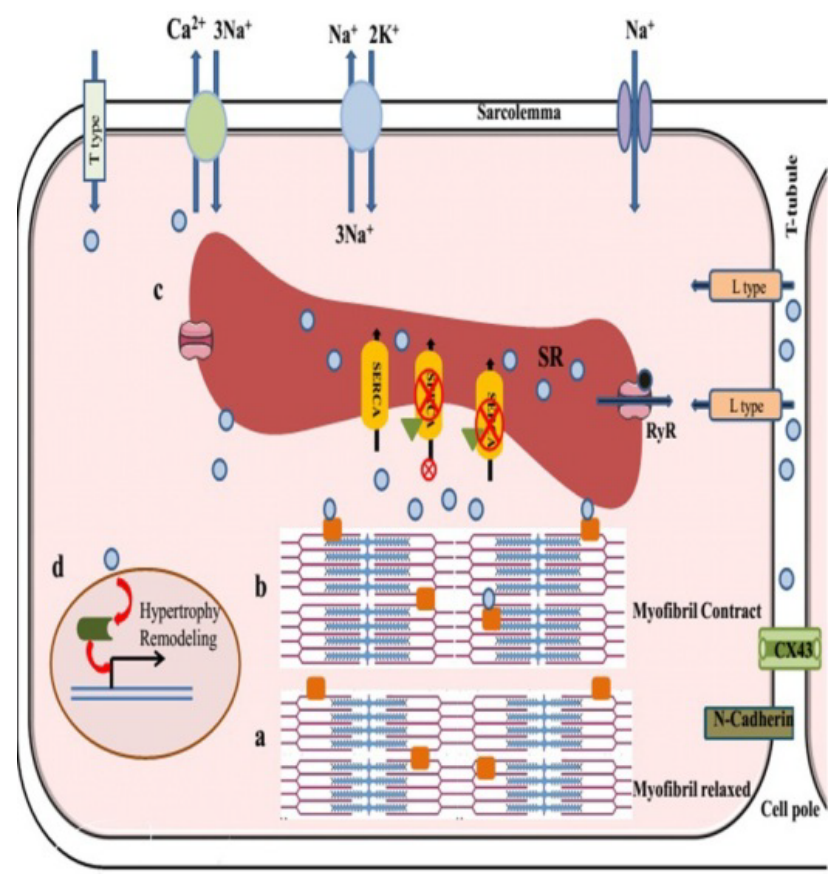

Figure 5: Schematic drawing depicting the effect of hyperglycemia on myofibrillar contraction. a. relaxed myofibril b. contracted myofibril c. sarcoplasmic reticulum d. nucleus. Calcium level is increased in the cytosol via ryanodine receptor (RyR) on SR membrane and $L$ type receptors present on sarcomere mediating myofibrillar contraction. This condition is reverted back by reuptake of calcium into SR via SERCA pump. Under hyperglycemic ambience glycated SERCA pump prevents the reuptake of calcium, thereby increasing the contraction of myocytes causing ventricular dysfunctioning. 
augment glucose transport. Thus decreased AMPK not only indicates decreased autophagy but also decreased muscle contratibility and glucose intake [92]. To combat the increased work load, ventricular remodeling occurs, resulting in cardiac hypertrophy that initially maintains cardiac output, but leads to diastolic and systolic dysfunction in later stages. The increased heart mass due to ventricular remodeling is marked by increased myocyte volume, without myocyte proliferation. The augmented expression of fetal cardiac genes has found to influence increased heart mass that has led to cardiac hypertrophy. Azelnidipine (AZL) has been examined not only in ameliorating oxidative stress and apoptosis, but also in maintaining $\mathrm{Ca}^{2+}$ homeostasis by normalizing SERCA2, ryanodine receptor (RyR) proteins and Troponin I expression, thereby increasing the of calcium clearance from cytosol [93].

It has been observed that in 2 months old adult female SpragueDawley Ivanovas rats, early stage of DCM (first week) is marked by subtle changes in myocyte morphology and contractibility, which in otherwise are interdependent. The decrease in heart weight of $25 \%$ is found to be positively correlated with decreased myocyte volume of $30 \%$ after 14 days of STZ injection [8]. This decrease in myocyte volume is found to be birectional, denoted by reduced length to width ratio, the reason for such volumetric change lies on specific anatomical changes in diabetic left ventricle.

\section{Microalbuminuria}

The mechanism of diabetic cadiomyopathy is multifactorial nominally myocyte dysfunction, caused by myocardial fibrosis, metabolic disturbances, microvascular diseases, autonomic dysfunction and insulin resistance. Microalbuminuria being the marker for renal microangiopathy and the severity of microangiopathy is related to degree of albuminuria, the mechanisms involved in microangiopathy progressing to myocardial dysfunction is gaining attention. Albeit, the exact relation between microalbuminuria and cardiomyopathy remains elusive, it has been speculated that the vascular changes that lead to renal dysfunction and cardiac dysfunction could be alike that, both are prone to similar alterations under disease condition. A combinatorial approach of angiotensin receptor blockers and peroxisome proliferatoractivated receptor (PPAR)-gamma agonist based treatment regimen has been postulated to mitigate the grimness of microalbuminuria and cardiac dysfunction. This has led to the hypothesis that renal and cardiac dysfunctioning may be an interdependent mechanism.

Recent findings have shown that cardiokines, as defined by Shimano et al. not only regulates the cardiac functioning and cardiac remodeling in pathological state, but also bridges the communication between heart and kidney (Figure 6) [94]. Such cardiokines include BNP (brain natriuretic peptide), ANP (atrial natriuretic peptide) and Follistatin-like 1 (Fstl1) also called TSC-36. As a supportive evidence for the hypothesis, the tissue specific secretion of Fstll has been found to have differential nephroprotective action.

Cardiac specific Fstl1 (cFstl1) has been proved to elevate AMPK expression that mitigates renal injury, by minifying the level of proinflammatory cytokine and NADPH oxidase mediated ROS generation in mesangial cells [95]. So, the progression of renal dysfunctioning is marked by impaired cardiokine secretion that indicates the initiation of cardiac dysfunctioning. Thence, cardiac dysfunctioning is speculated to occur early in the course of renal failure. This is in agreement with the prospective multicentre observational studies (GREECS, GRACE), where in creatinine clearance has been found to be a vital predictor of hospital mortality and major bleeding episodes in patients admitted with acute coronary syndrome diagnosis $[96,97]$.

Consistent with these reports are the findings on GDF15 (Growth differentiation factor - 15) a member of TGF- $\beta$ superfamily that is expressed in liver and placenta at baseline, but poorly expressed in adult heart under physiological condition. GDF-15 has been induced with the onset of acute kidney injury (AKI) that is positively correlated with $\mathrm{N}-\mathrm{GAL}$, a kidney injury marker and is found to exacerbate glomerular filtration rate (GFR). However, cardiokine Fstl1 has been demonstrated to induce GDF15 in myocardial infarction $[98,99]$. In contrast, Xu et al. has reported that, in neonatal cardiomyocytes and in adult mice, GDF15 induced under injury alleviates cardiac hypertrophy and dilated cardiomyopathy via smad $2 / 3$ mediated AKT activation, which is akin to TGF- $\beta$ signaling [100]. Thence, whilst, cardiokines ameliorate the pathological condition, its activity should be concentration dependent that, augmented secretion may exacerbate the damage.

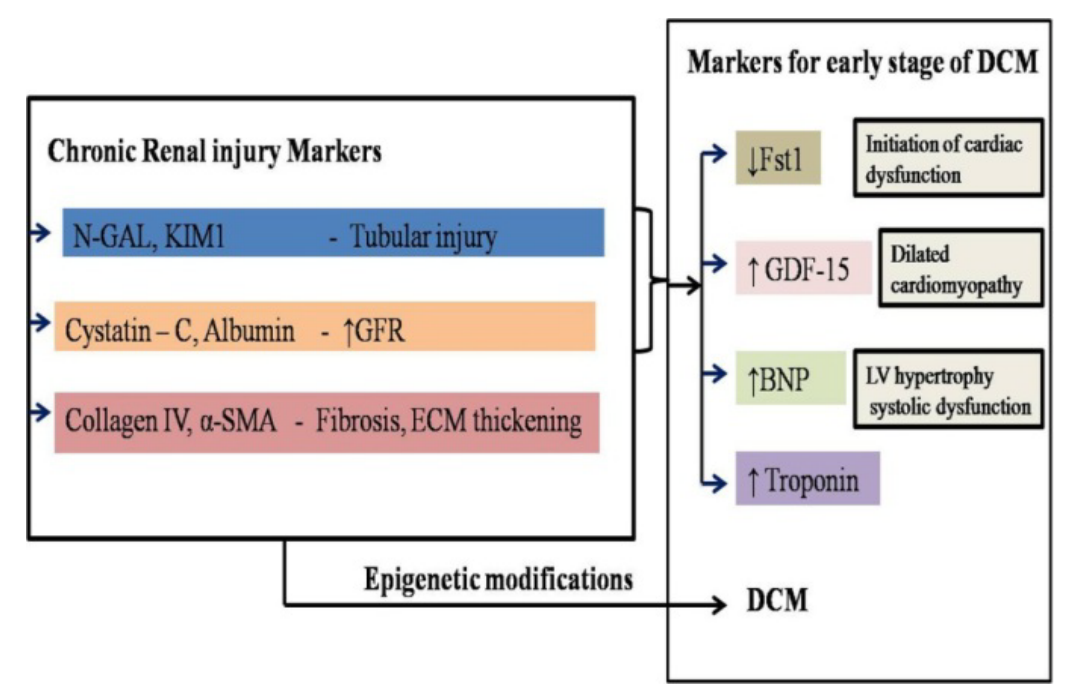

Figure 6: Schematic representing the interrelationship between diabetic mediated renal injury and early stage of diabetic cardiomyopathy. Cardiokines with altered expression at the stage of impaired glomerular filtration and tubular injury is linked to incidence of diabetic cardiomyopathy. Furthur epigenetic modifications observed under renal injury also influences DCM. 
In addition certain peptides and proteins have been identified as a marker for diabetic cardiomyopathy that facilitates the understanding of interrelation between heart and renal functioning. Such markers include troponin a calcium dependent cardiac specific actomyosinATPase inhibitory protein, involved in actin-myosin interaction and muscle relaxation, and B-type natriuretic peptide (BNP), a 32-amino acid peptide released by ventricular cardiac myocytes in response to wall stress. The isoforms of troponin expressed in heart includes troponin $\mathrm{I}(\mathrm{cTnI})$, troponin $\mathrm{T}(\mathrm{cTnT})$ and troponin $\mathrm{C}(\mathrm{cTnC})$.

Both these markers exert its effect by binding to natriuretic peptide receptor (NPR-A) [101]. It has been postulated that renal artery denervation (RAD) reduced the cardio-metabolic abnormalities. The reduced sympathetic activation due to RAD led to decreased catecholamine release that decreased the blood pressure and wall stress mitigating ventricular dysfunction. This is in agreement with the decreased pro-type B natriuretic peptide (proBNP) [102,103]. However, in fetus of diabetic mother, the augmented proBNP and cTnT has been implicated to prevent cardiac hypertrophy.

Further, increased NT-proBNP substantiated chronic kidney disease with asymptomatic LV hypertrophy. Subsequently the next stage in diabetic cardiomyopathy marked by systolic dysfunction, LV dilation and higher LV filling pressure develops, and is characterized by elevated troponin level (cTnT) with concomitant increase in NTproBNP. These cardiac functional abnormalities have been observed in a prospective observational study, where in diabetic patients in end stage renal failure (ESRD) undergoing dialysis had augmented cTnT and NT-proBNP [104]. So it can be concluded that the proteins and cardiokines once functional in preventing cardiac injury in fetus, may turn deleterious in adult heart upon increased secretion, or the cardiokines secreted remains ineffective to combat the damage.

\section{Epigenetics of Diabetic Cardiomyopathy}

Epigenetics described as a non-genetic change in gene expression with accompanied mitotic and meiotic inheritance and without any altered nucleotide sequence. Several environmental factors influence chromatin rearrangements implicating DNA methylation, histone post-translational modification and chromatin remodeling [105,106].
Post translational modifications of histones include both permissive and repressive modifications. Though the epigenetics of diabetic cardiomyopathy remains elusive, renal failure influencing DCM is associated with epigenetic modifications that activate or suppress transcription (Table 1). Recent study has shown that, in hearts of diabetic mice, elevated $\mathrm{H} 3$ acetylation at lysine 9 (H3K9Ac9) and 23 (H3K9Ac23), H3 dimethylation at lysine 4 (H3K4me2) and 9 (H3K9me2), and $\mathrm{H} 3$ phosphorylation at serine 10 were observed. Uninephrectomized $\mathrm{db} / \mathrm{db}$ mice have further augmented these histone modifications along with decreased $\mathrm{H} 3$ dimethylation at lysine 9 (H3K9me2). These histone modification under renal failure has been associated with increased expression of cardiomyopathy-related genes involved in tissue remodeling that includes myosin heavy chains 3 , 6 , and 7 , myosin light chain 3 , as well as tubulin- $\alpha$, catenin- $\alpha 1$, and laminin- $\beta 2$ [107]. Consistent with these findings, angiotensin II type 1 inhibitor losartan has been substantiated to ameliorate diabetic nephropathy, concomitant with elevation of $\mathrm{H} 3 \mathrm{~K} 9$ and $\mathrm{H} 3 \mathrm{~K} 14$ acetylation implicated in DCM .

$\mathrm{H} 3$ phosphorylation of serine 10 is found to be regulated by calcium/ calmodulin-dependent protein kinase II $\boldsymbol{\delta}$ (CaMKII $\boldsymbol{\delta}$ ). Its utility in fetal cardiac development turns deleterious under hemodynamic stress, which is critical for cardiac hypertrophy [108].

Augmented H2AK119 and H2BK120 ubiquitination has been positively correlated with $\mathrm{H} 3 \mathrm{~K} 4 \mathrm{me} 1, \mathrm{H} 3 \mathrm{~K} 4 \mathrm{me} 2$ and $\mathrm{H} 3 \mathrm{~K} 4 \mathrm{me} 3$ levels at the promoter of fibrotic genes involved in diabetic nephropathy progression. Along with these PTM, Kadakol et al. has demonstrated an increased H3K9me2, H3K36me2, H3K79me2, H3T3phospho, $\mathrm{H} 3 \mathrm{~K} 27 \mathrm{Ac}$ and $\mathrm{H} 3 \mathrm{~K} 56 \mathrm{Ac}$ in type 2 diabetic rats progression to DCM. The cardioprotective role of esculetin has been demonstrated by decreased $\mathrm{AT}_{1} \mathrm{R}$ and $\mathrm{AT}_{2} \mathrm{R}$ expression and increased ACE2 expression. Hence, the histone ubiquitination of $\mathrm{H} 2 \mathrm{~A} / \mathrm{H} 2 \mathrm{~B}$ should have been occurred in the promoter region of $\mathrm{AT}_{1} \mathrm{R}, \mathrm{AT}_{2} \mathrm{R}$ and $\mathrm{ACE} 2$ that facilitated an increased expression of genes involved diabetic cardiomyopathy progression [109].

Further activation of ATP dependent chromatin remodeling proteins (CRP) has been substantiated in DCM. Among 4 CRPs, four different SWI/SNF (switching defective/sucrose non-fermenting)

\begin{tabular}{|c|c|c|c|c|}
\hline \multicolumn{5}{|l|}{ Histone modification } \\
\hline Source & PTM & Drugs & Role of drugs & Promoter \\
\hline H3K9/14 & $\uparrow$ Acetylation & Losartan & Inhibits HAT & RAGE and PAI-1 \\
\hline $\begin{array}{l}\mathrm{H} 3 \mathrm{~K} 4 \\
\mathrm{H} 3 \mathrm{~K} 9 / 14\end{array}$ & $\begin{array}{l}\downarrow \text { Methylation } \\
\downarrow \text { Acetylation }\end{array}$ & $\begin{array}{l}\text { Esculetin (6, } \\
\text { 7-dihydroxycoumarin) }\end{array}$ & $\begin{array}{l}\text { Radical scavenger Anti-proliferative } \\
\text { Anti-fibrotic }\end{array}$ & TGF- $\beta$, PPAR- $\gamma$ \\
\hline H3K36 & $\uparrow$ Methylation & Losartan & Inhibits methyl transferase & RAGE and PAI-1 \\
\hline H3K9/14 & $\uparrow$ Acetylation & Atorvastatin & Promotes HAT & ACE2 \\
\hline H3K4/14 & $\downarrow$ Acetylation & Curcumin analogue C66 & p300/CBP-mediated deacetylation & - \\
\hline $\mathrm{H} 2 \mathrm{AK} 119$ and $\mathrm{H} 2 \mathrm{BK} 120$ & $\uparrow$ ubiquitination & Esculetin & $\begin{array}{l}\text { Reversal of post translational } \\
\text { modifications }\end{array}$ & $\underset{1}{\mathrm{AT}} \mathrm{R}, \underset{2}{\mathrm{AT}} \mathrm{R}$ and ACE2 \\
\hline $\mathrm{H} 3 \mathrm{~K} 27$ and $\mathrm{H} 3 \mathrm{~K} 9$ & $\downarrow$ Trimethylation & - & - & ANP, BNP \\
\hline \multicolumn{5}{|l|}{ DNA methylation } \\
\hline Source & PTM & Drugs & Role of drugs & Promoter \\
\hline CpG islands & Demethylation & - & - & Keap1 \\
\hline CpG islands & $\uparrow$ Methylation & SPV106 & HAT activator & Cell cycle and DNA repair \\
\hline \multicolumn{5}{|l|}{ Chromatin remodeling } \\
\hline Source & \multicolumn{2}{|l|}{ PTM } & Effect of PTM & Promoter \\
\hline Histone deacetylation & \multicolumn{2}{|c|}{$\uparrow \mathrm{Brg} 1, \uparrow \mathrm{Baf} 180$ and $\uparrow \mathrm{Baf60c}$} & Diabetic cardiac hypertrophy & Fetal gene \\
\hline Ser727 and Try705 & \multicolumn{2}{|c|}{ Phosphorylation, $\downarrow$ Brg1 } & Diastolic dysfunction & STAT3 \\
\hline
\end{tabular}

Table 1: Epigenetic modifications involved in diabetic cardiomyopathy. 
subfamilies have been investigated to play a vital role in heart development, in which SWI/SNF complexes is solely involved in adult cardiomyocytes. Brahma-associated factor (BAF) complex, the mammalian orthologue of the SWI/SNF complex consists of $14 \mathrm{BAF}$ subunits, which contain either brahma (Brm) or brahma-related gene 1 (Brg1) as the ATPase subunit. Brg1, which is involved in fetal cardiac development has been overexpressed in adult cardomyocytes under cardiac stress, that interacts with HDACs and PARP1, and its activation is marked by shift in MHC from $\alpha-\mathrm{MHC}$ to embryonic $\beta$-MHC, an initiator of hypertrophy $[110,111]$. In high salt fed hypertensive rats Brg1, Baf180, and Baf60c have found to be enriched in promoters of fetal genes ANP and BNP, which might be due to deacetylation of histones in these promoter regions.

In contrast $\mathrm{Xu}$ et al. has shown that $\mathrm{Brg} 1$ is downregulated in the early stage of type 1 diabetes concomitant with decreased HO1 and phosphorylated Ser727 and Try705 in STAT3, marked by diastolic dysfunction [112]. Further adiponectin has been found to increase $\mathrm{HO} 1$ by $\mathrm{Nrf} 2$ (Nuclear factor-erythroid-2-related factor-2) mediated Brg1 activation, which in turn recruits RNA polymerase II [113]. Hence PTM and chromatin remodeling are conceived to be a codependent mechanism and the drugs targeting these modifications may combat both renal failure and cardiovascular disease. In addition DNA methylation has been found to regulate the progression of DCM. In diabetic subjects, Keap1 (Keltch-like ECH-associated protein 1) is found to be highly transcribed due to demethylation of $\mathrm{CpG}$ islands in keap1 promoter that mediates proteosomal degradation of $\mathrm{Nrf} 2$, blocking anti-oxidant mechanism [114].

Vecellio et al. has shown that in type 2 diabetes induced cardiac mesenchymal stem cells, the CpG islands of gene promoters involved in cell cycle and DNA repair (TP53, BRCA1 and BRCA2, CCNB1, CCNF, CDK2, MCM2, and RAD5) has been hypermethylated that is associated with hypoacetylation of $\mathrm{H} 3 \mathrm{~K} 9$ and trimethylation of $\mathrm{H} 3 \mathrm{~K} 27$, a condition observed in DCM. These observations were in parallel with down regulation of $\mathrm{p} 300 / \mathrm{CBP}$-associated factor (PCAF), its isoform 5-a (GCN5a) and GCN5-related N-acetyltransferases (GNAT). SPV106, a histone acetyl transferase has found to attenuate these modifications. Thus acetylation of histones prevents open access of DNA for methylation, promoting demethylation of promoters [115].

Since these epigenetic changes form the basis for origin and progression of DCM, interventions should not only target in attenuating the signaling cascades but also in ameliorating these epigenetic modifications.

\section{Glycemic Excursion, Family History and Diet: Forgotten Truth in CVD}

The vascular complications as discussed above are apparent in diabetic cases that are unmonitored, and the glycemic disorders in diabetics not only include hyperglycemia but also the intra-day glycemic excursions and its rate of excursion, which is not considered in evaluating the treatment strategies [116]. The rate of glycemic excursion varies based on the carbohydrate content in the diet, as listed in the glycemic index (GI). The high GI diet is found to elevate the blood glucose level compared to the low GI diet, and the post prandial glucose level of high GI diet in the day time is found to cause a significant impact on glycemic variability.

Numerous epidemiological studies on diabetes based cardiovascular diseases focused on hyperglycemia. In Diabetes Control and Complications Trial (DCCT) study, the glycosylated hemoglobin has been manifested to be strongly associated with cardiovascular disease (CVD) in type 1 diabetics and the EDIC study substantiates that the major risk factor of CVD is microalbuminuria and albuminuria [117]. According to accord study even very tight control of blood glucose level failed to control cardiovascular diseases. Thus even though, glycosylated hemoglobin (HbAlc) is widely considered to be a marker for chronic hyperglycemia, it is not the only criteria to be considered in macrovascular complications, especially when cardiovascular disease is concerned. Consistent with this fact is the evidence given by a comparative epidemiological study which has proved that glycemic disorders are observed in diabetic patients with coronary artery disease (CAD) but not in non CADs $[118,119]$.

Thus transient glucose level would be a major common cause for persistent effects in CVD which is independent of HbAlc, which failed to explicate this effect. Glycated albumin (GA) represents the glycemic excursion and post-prandial hyperglycemia. Since faster glycation of albumin reduces its anti-oxidant potential, it is found to be the major contributor of endothelial dysfunction and mitochondrial reactive oxygen species (ROS) formation, the major cause for CVD. The reduced antioxidant potential of glycated albumin is found to be the initiator of ROS production. The major Intensive glucose treatment studies have shown that it did not have beneficial effect on the mortality of macrovascular complications; this might be due to the counterbalancing effect of severe hypoglycemia [120].

One such severity of transient hyperglycemia includes formation of AGE induced metabolic memory. Not only the extracellular and blood plasma are susceptible to glycation, but also the intracellular products especially the mitochondrial proteins and mitochondrial DNA. The short fluctuating spikes of glycemia exacerbated mitochondrial damage, reactive oxygen species formation and nitrotyrosine (NT) level, compared to chronic hyperglycemia. These accentuated metabolic memory observed under intermittent normoglycemia as the vital factor to be considered in diabetics [121].

Thus not only hyperglycemia increases the NT level but also the post prandial hypertriglyceridemia ( $\mathrm{ppHTG),} \mathrm{which} \mathrm{is} \mathrm{substantiated} \mathrm{to}$ be an independent predictor of CVD, evidenced by increased intimamedia thickness of carotid artery (IMT) in type 2 diabetics [122-124]. Ceriello et al. has reported that the endothelial dysfunction driven by oscillating blood glucose is related to increased 3-nitroglycerine level, a marker of reactive oxygen species and 8-iso prostaglandin F2a (PGF2 $\alpha$ ) an indicator of lipid peroxidation [125]. In endothelial cells of umbilical cord, an increase in IL- 6 and adhesion molecules ICAM-1 and VCAM-1 has been monitored, which is influenced by oxidative stress mediated PARP (polyADP ribose polymerase) activation and isoforms of PKC $[126,127]$. Further, the increased nitrotyrosine and peroxynitrite irreversibly oxidized the cysteine residue in SERCA, blocking calcium reuptake.

Thus, primarily, while CVD is concerned, the severity of glycemic fluctuations should be considered to frame an effective treatment strategy. Even studies on diabetic cardiomyopathy centralizes only on chronic hyperglycemia, and the treatment strategies concentrates mainly on lowering lipid level, which has been ascertained to fluctuate the blood glucose level. So far, none of the treatment regimen has been demonstrated to influence long term normoglycemia $[128,129]$.

Secondarily, family history of the patients also influences CVD incidence. Pannacciulli et al. has demonstrated that the first degree relatives of T2DM cases along with slight increase in blood glucose augmented IMT at an early stage of diabetes. Their post prandial blood 
glucose level has been found to be relatively higher than in patients without genetic predisposition, which might be due to the altered substrate metabolism and decreased incretin hormones secretion that influence post prandial insulin secretion [130,131]. Eventually, Irrespective of age, obese individuals are more prone to endothelial dysfunction. Further, diet plays a vital role in management of obesity mediated T2DM conditions and its related complications. Ciccone et al. has reported that dietary supplementation of Omega-3 Polyunsaturated Fatty Acids in obese children has decreased inflammatory cytokine production and improved endothelial functioning [132]. Thence, when treatment for CVD is concerned, awareness on dietary habits that mitigate the severity of CVD among diabetic patients may help avoid obesity related complications and the detrimental effects of transient hyperglycemia.

\section{Conclusion}

Recent evidences support the fact that DCM is initiated via metabolic perturbations in myocytes, in response to hyperglycemic milieu. Chronic hyperglycemia intends to cause oxidative stress, ER stress, apoptosis and fibrosis inducing structural disturbances and functional abnormalities. Though DCM has been reported in patients progressing with microangiopathy, there remains a considerable controversy over what are the actual underlying mechanisms of such coexistence. This review relates one of such mechanism as, epigenetic changes that occur along with renal failure, though this area has to be explored more to elucidate the codependence. Despite the understanding on chronic hyperglycemia mediated DCM, fluctuating blood glucose level as seen in diabetic patients due to post prandial glucose level and medications should also be considered, to maintain long term normoglycemia that could combat the detrimental effects of transient HG. However family history and obesity regardless of age factor has also been found to influence CVD incidence. Besides, this review conveys that, the molecular mechanisms cardiac myocytes carry out under chronic HG is conceived to be adaptive in the earlier stages, which turns out to be maladaptive influencing disease progression. Thus in diabetic patients family history and obesity should be considered to decide the time for intervention, and the treatment strategies focusing the early stage of DCM considering the epigenetic changes and glycemic excursions may provide a better management of DCM progression.

\section{Acknowledgement}

Authors are also thankful to VIT University for providing the resources to prepare this review.

\section{Conflict of Interest}

The authors declare that there was no conflict of interest.

\section{References}

1. Pappachan JM, Varughese GI, Sriraman R, Arunagirinathan G (2013) Diabetic cardiomyopathy: Pathophysiology, diagnostic evaluation and management. World J Diabetes 4: 177-189.

2. Rubler S, Dlugash J, Yuceoglu YZ, Kumral T, Branwood AW, et al. (1972) New type of cardiomyopathy associated with diabetic glomerulosclerosis. Am J Cardiol 30960: 595-602.

3. Picatoste B, Ramírez E, Caro-Vadillo A, Iborra C, Ares-Carrasco S, et al. (2013) Sitagliptin Reduces Cardiac Apoptosis, Hypertrophy and Fibrosis Primarily by Insulin-Dependent Mechanisms in Experimental type-II Diabetes Potential Roles of GLP-1 Isoforms. PLoS One 8: e78330.

4. Wang L, Li J, Li D (2015) Losartan reduces myocardial interstitial fibrosis in diabetic cardiomyopathy rats by inhibiting JAK / STAT signaling pathway. Int J Clin Exp Pathol 8: 466-473.

5. Yu HT, Zhen J, Pang B, Gu JN, Wu SS (2015) Ginsenoside Rg1 ameliorates oxidative stress and myocardial apoptosis in streptozotocin-induced diabetic rats. J Zhejiang Univ Sci B 16: 344-354.

6. Dyntar D, Sergeev P, Klisic J, Ambühl P, Schaub M C, et al. (2006) High glucose alters cardiomyocyte contacts and inhibits myofibrillar formation. J Clin Endocrinol Metab 91: 1961-1967.

7. Ramírez E, Klett-Mingo M, Ares-Carrasco S, Picatoste B, Ferrarini A, et al. (2013) Eplerenone attenuated cardiac steatosis, apoptosis and diastolic dysfunction in experimental type-II diabetes. Cardiovasc Diabetol 12: 172.

8. Dhalla NS, Rangi S, Zieroth S, Xu Y J (2012) Alterations in sarcoplasmic reticulum and mitochondrial functions in diabetic cardiomyopathy. Exp Clin Cardiol 17: 115-120.

9. Amaral N, Okonko D O (2015) Metabolic abnormalities of the heart in type II diabetes. Diabetes Vasc Dis Res 12: 239-248.

10. Shim CY, Song BW, Cha MJ, Hwang KC, Park S, et al. (2014) Combination of a peroxisome proliferator-activated receptor-gamma agonist and an angiotensin II receptor blocker attenuates myocardial fibrosis and dysfunction in type 2 diabetic rats. J Diabetes Investig 5: 362-371.

11. Patil VC, Patil HV, Shah KB, Vasani JD, Shetty P (2011) Diastolic dysfunction in asymptomatic type 2 diabetes mellitus with normal systolic function. $J$ Cardiovasc Dis Res 2: 213-222.

12. Lam CS (2015) Diabetic cardiomyopathy: An expression of stage B heart failure with preserved ejection fraction. Diabetes Vasc Dis Res 12: 234-238.

13. Ernande L, Derumeaux G (2012) Diabetic cardiomyopathy: Myth or reality? Arch. Cardiovasc Dis 105: 218-225

14. Falcão-Pires I, Leite-Moreira A F (2012) Diabetic cardiomyopathy: Understanding the molecular and cellular basis to progress in diagnosis and treatment. Heart Fail Rev 17: 325-344.

15. Nagueh SF, Appleton CP, Gillebert TC, Marino PN, Oh JK, et al. (2009) Recommendations for the evaluation of left ventricular diastolic function by echocardiography. Eur J Echocardiogr 10: 165-193.

16. Norton JM (2001) Toward consistent definitions for preload and afterload. Adv Physiol Educ 25: 53-61.

17. Widya RL, van der Meer RW, Smit JW, Rijzewijk LJ, Diamant M, et al. (2013) Right ventricular involvement in diabetic cardiomyopathy. Diabetes Care 36: 457-462.

18. Qian C, Gong L, Yang Z, Chen W, Chen Y, et al. (2015) Diastolic dysfunction in spontaneous type 2 diabetes rhesus monkeys: a study using echocardiography and magnetic resonance imaging. BMC Cardiovasc Disord 15: 59.

19. Khouri SJ, Maly GT, Suh DD, Walsh TE (2004) A practical approach to the echocardiographic evaluation of diastolic function. J Am Soc Echocardiogr 17: 290-297.

20. Bella JN, Palmieri V, Roman MJ, Liu JE, Welty TK, et al. (2002) Mitral ratio of peak early to late diastolic filling velocity as a predictor of mortality in middleaged and elderly adults: The strong heart study. Circulation 105: 1928-1933.

21. Galderisi M (2006) Diastolic Dysfunction and Diabetic Cardiomyopathy Evaluation by Doppler Echocardiography. J Am Coll Cardiol 48: 1548-1551.

22. Nikitin NP, Witte KK, Ingle L, Clark AL, Farnsworth TA, et al. (2005) Longitudinal myocardial dysfunction in healthy older subjects as a manifestation of cardiac ageing. Age Ageing 34: 343-349.

23. Koyama J, Ray-Sequin PA, Falk RH (2003) Longitudinal myocardia function assessed by tissue velocity, strain, and strain rate tissue doppler echocardiography in patients with $\mathrm{AL}$ (primary) cardiac amyloidosis. Circulation 107: 2446-2452

24. Zhang H, Wei Z, Zhu X, Li H, Yu M, et al. (2014) Assessment of Left Ventricular Myocardial Systolic Acceleration in Diabetic Rats Using Velocity Vector Imaging. J Ultrasound Med 33: 875-883.

25. Cioffi G, Faganello G, De Feo S, Berlinghieri N, Tarantini L, et al. (2013) Combined circumferential and longitudinal left ventricular systolic dysfunction in patients with type 2 diabetes mellitus without myocardial ischemia. Exp Clin Cardiol 18: e26-31.

26. Ernande L, Rietzschel ER, Bergerot C, De Buyzere ML, Schnell F, et al. (2012) Impaired myocardial radial function in asymptomatic patients with type 2 diabetes mellitus: A speckle-tracking imaging study. J Am Soc Echocardiogr 23: 1266-1272. 
Citation: Muralidaran Y, Viswanathan P (2015) Diabetic Cardiomyopathy: A New Perspective of Mechanistic Approach. J Diabetes Metab 6: 605. doi:10.4172/2155-6156.1000605

Page 13 of 15

27. Jaswal JS, Keung W, Wang W, Ussher JR, Lopaschuk GD (2011) Targeting fatty acid and carbohydrate oxidation - A novel therapeutic intervention in the ischemic and failing heart. Biochim Biophys Acta - Mol Cell Res 1813: 13331350

28. Abel ED (2005) Metabolic perturbations in the diabetic heart: Mechanisms and molecular targets. Drug Discov Today Dis Mech 2: 115-122.

29. Carley AN, Severson DL (2005) Fatty acid metabolism is enhanced in type 2 diabetic hearts. Biochim Biophys Acta 1734: 112-126.

30. Finck BN, Han X, Courtois M, Aimond F, Nerbonne JM, et al. (2003) A critica role for PPARalpha-mediated lipotoxicity in the pathogenesis of diabetic cardiomyopathy: modulation by dietary fat content. Proc Natl Acad Sci U S A 100: 1226-31.

31. Talati M, Hemnes A (2015) Fatty acid metabolism in pulmonary arteria hypertension : role in right ventricular dysfunction and hypertrophy. pulm circ 5: 269-278.

32. Luiken JJ, Koonen DP, Willems J, Zorzano A, Becker C, et al (2002) Insulin Stimulates Long-Chain Fatty Acid Utilization by Rat Cardiac Myocytes Through Cellular Redistribution of FAT/CD36. Diabetes 51: 3113-3119.

33. Yang J, Sambandam N, Han X, Gross RW, Courtois M, et al. (2007) CD36 Deficiency Rescues Lipotoxic Cardiomyopathy. Circ Res 100: 1208 -1217.

34. Burkart EM, Sambandam N, Han X, Gross R W, Courtois M, et al. (2007) Nuclear receptors PPAR $\beta$ / $\delta$ and PPAR $\alpha$ direct distinct metabolic regulatory programs in the mouse heart. $\mathrm{J}$ clin invest 117: 3930-3939.

35. Chen R, Liang F, Morimoto S, Li Q, Moriya J, et al. (2010) The Effects of a PPAR agonist on myocardial damage in obese diabetic mice with heart failure. Int Heart J 51: 199-206.

36. Bugger H, Guzman C, Zechner C, Palmeri M, Russell KS, et al. (2011) Uncoupling protein downregulation in doxorubicin-induced heart failure improves mitochondrial coupling but increases reactive oxygen species generation. Cancer Chemothe Pharmacol 67: 1381-1388.

37. Taegtmeyer H, Lubrano G (2014) Rethinking cardiac metabolism: metabolic cycles to refuel and rebuild the failing heart. F1000Prime Rep 6: 90

38. Xu J, Zhou Q, Xu W, Cai L (2012) Endoplasmic reticulum stress and diabetic cardiomyopathy. Exp Diabetes Res 2012: 827971.

39. Li Z, Zhang T, Dai H, Liu G, Wang H, et al. (2007) Involvement of endoplasmic reticulum stress in myocardial apoptosis of streptozocin-induced diabetic rats. $\mathrm{J}$ Clin Biochem Nutr 41: 58-67.

40. Nyengaard JR, Ido Y, Kilo C, Williamson JR (2004) Interactions between hyperglycemia and hypoxia: implications for diabetic retinopathy. Diabetes 53: 2931-2938.

41. Singh F, Charles AL, Schlagowski Al, Bouitbir J, Bonifacio A, et al. (2015) Reductive stress impairs myoblasts mitochondrial function and triggers mitochondrial hormesis. Biochim Biophys Acta, 1853: 1574-1585.

42. Zhang C, Huang C, Tian Y, Li X (2014) Polyol pathway exacerbated ischemia/ reperfusion-induced injury in steatotic liver. Oxid Med Cell Longev 2014: 963629 .

43. Kanwar YS, Sun L, Xie P, Liu FY, Chen S (2011) A glimpse of various pathogenetic mechanisms of diabetic nephropathy. Annu Rev Pathol 6: 395423.

44. Waller AP, George M, Kalyanasundaram A, Kang C, Periasamy M, et al. (2013) GLUT12 functions as a basal and insulin-independent glucose transporter in the heart. Biochim Biophys Acta 1832: 121-127.

45. Ebeling P, Koistinen HA, Koivisto VA (1998) Insulin-independent glucose transport regulates insulin sensitivity. FEBS Lett 436: 301-303.

46. Tang WH, Cheng WT, Kravtsov GM, Tong XY, Hou XY, et al. (2010) Cardiac contractile dysfunction during acute hyperglycemia due to impairment of SERCA by polyol pathway-mediated oxidative stress. Am J Physiol Cell Physiol 299: C643-653.

47. Yan LJ (2014) Pathogenesis of chronic hyperglycemia: from reductive stress to oxidative stress. J Diabetes Res 2014: 137919

48. Tiwari AK, Kumar DA, Pisupati S, Sweeya H, Chauhan AV, et al. (2014) Vegetables' juice influences polyol pathway by multiple mechanisms in favour of reducing development of oxidative stress and resultant diabetic complications. Pharmacogn Mag, 10: S383-391.
49. Galvez AS, Ulloa JA, Chiong M, Criollo A, Eisner V, et al. (2003) Aldose reductase induced by hyperosmotic stress mediates cardiomyocyte apoptosis Differential effects of sorbitol and mannitol. J Biol Chem 278: 38484-38494.

50. Rajamani U, Essop MF (2010) Hyperglycemia-mediated activation of the hexosamine biosynthetic pathway results in myocardial apoptosis. Am J Physiol Cell Physiol 299: C139-147.

51. Zeidan Q, Hart GW (2010) The intersections between O-GlcNAcylation and phosphorylation: implications for multiple signaling Pathways. J Cell Sci 123 13-22.

52. Marsh SA, Dell'Italia LJ, Chatham JC (2011) Activation of the hexosamine biosynthesis pathway and protein O-GIcNAcylation modulate hypertrophic and cell signaling pathways in cardiomyocytes from diabetic mice. Amino Acids 40 819-828.

53. Wollaston-Hayden EE, Harris RB, Liu B, Bridger R, Xu Y, et al. (2015) Global O-GlcNAc Levels Modulate Transcription of the Adipocyte Secretome during Chronic Insulin Resistance. Front Endocrinol (Lausanne) 5: 223.

54. Zimmerman AD, Harris RB (2015) In vivo and in vitro evidence that chronic activation of the hexosamine biosynthetic pathway interferes with leptindependent STAT3 phosphorylation. Am J Physiol Regul Integr Comp Physio 308: R543-555.

55. Majumdar G, Harmon A, Candelaria R, Martinez-Hernandez A, Raghow R, et al. (2003) O-glycosylation of Sp1 and transcriptional regulation of the calmodulin gene by insulin and glucagon. Am J Physiol Endocrinol Metab 285: E584-591.

56. Belke DD, Betuing S, Tuttle MJ, Graveleau C, Young ME, et al. (2002) Insulin signaling coordinately regulates cardiac size, metabolism, and contractile protein isoform expression. J Clin Invest 109: 629-639.

57. Laczy B, Fülöp N, Onay-Besikci A, Des Rosiers C, Chatham J C (2011) Acute regulation of cardiac metabolism by the hexosamine biosynthesis pathway and protein O-GIcNAcylation. PLoS One 6: e18417.

58. Beleznai T, Bagi Z (2012) Activation of hexosamine pathway impairs nitric oxide (NO)-dependent arteriolar dilations by increased protein O-GIcNAcylation. Vascul Pharmacol 56: 115-121

59. Lunde IG, Aronsen JM, Kvaløy H, Qvigstad E, Sjaastad I, et al. (2012) Cardiac $\mathrm{O}-\mathrm{GlcNAc}$ signaling is increased in hypertrophy and heart failure. Physio Genomics 44: 162-172.

60. Bodiga VL, Eda SR, Bodiga S (2014) Advanced glycation end products: role in pathology of diabetic cardiomyopathy. Heart Fail Rev 19: 49-63.

61. Candido R, Forbes J M, Thomas M C, Thallas V, Dean R G, et al. (2003) A breaker of advanced glycation end products attenuates diabetes-induced myocardial structural changes. Circ Res 92: 785-792.

62. Kain V, Kumar S, Sitasawad SL (2011) Azelnidipine prevents cardiac dysfunction in streptozotocin-diabetic rats by reducing intracellular calcium accumulation, oxidative stress and apoptosis. Cardiovasc Diabetol 10: 97.

63. Choi SW, Song JK, Yim YS, Yun HG, Chun KH (2015) Glucose Deprivation Triggers Protein Kinase C-dependent $\beta$-Catenin Proteasomal Degradatio. J Biol Chem 290: 9863-9873.

64. Marquez RT, Xu L (2012) Bcl-2:Beclin 1 complex: multiple, mechanisms regulating autophagy/apoptosis toggle switch. Am J Cancer Res 2: 214-221.

65. Kobayashi S, Liang Q (2015) Autophagy and mitophagy in diabetic cardiomyopathy. Biochim Biophys Acta 1852: 252-261.

66. Lee Y, Hong Y, Lee SR, Chang KT, Hong Y (2012) Autophagy contributes to retardation of cardiac growth in diabetic rats. Lab Anim Res 28: 99-107.

67. Xie Z, Lau K, Eby B, Lozano P, He C, et al. (2011) Improvement of cardiac functions by chronic metformin treatment is associated with enhanced cardiac autophagy in diabetic OVE26 mice. Diabetes 60: 1770-1778.

68. Marsh S A, Powell PC, Dell'italia LJ, Chatham JC (2013) Cardiac O-GIcNAcylation blunts autophagic signaling in the diabetic heart. Life Sci 92: 648-656.

69. Tang SW, Ducroux A, Jeang KT, Neuveut C (2012) Impact of cellular autophagy on viruses: Insights from hepatitis $B$ virus and human retroviruses. J Biomed Sci 19: 92.

70. Gonzalez CD, Lee MS, Marchetti P, Pietropaolo M, Towns R, et al. (2011) The emerging role of autophagy in the pathophysiology of diabetes mellitus. Autophagy 7: 2-11. 
Citation: Muralidaran Y, Viswanathan P (2015) Diabetic Cardiomyopathy: A New Perspective of Mechanistic Approach. J Diabetes Metab 6: 605. doi:10.4172/2155-6156.1000605

Page 14 of 15

71. Zou MH, Xie Z (2013) Regulation of interplay between autophagy and apoptosis in the diabetic heart New role of AMPK. Autophagy 9: 624-625.

72. He C, Zhu H, Li H, Zou MH, Xie Z (2013) Dissociation of Bcl-2-Beclin1 complex by activated AMPK enhances cardiac autophagy and protects against cardiomyocyte apoptosis in diabetes. Diabetes 62: 1270-1281.

73. Wang B, Yang Q, Sun YY, Xing YF, Wang YB, et al. (2014) Resveratrolenhanced autophagic flux ameliorates myocardial oxidative stress injury in diabetic mice. J Cell Mol Med 18: 1599-15611.

74. Lizbeth M, Flores J, Briones SL, Maciste H, Cervantes M, et al. (2014) A PPARY, NF-KB and AMPK-Dependent Mechanism May Be Involved in the Beneficial Effects of Curcumin in the Diabetic db/db Mice Liver. Molecules 19: 8289-8302.

75. Tang Y, Li M, Wang Y L, Threadgill M D, Xiao M, et al. (2015) ART1 promotes starvation-induced autophagy : a possible protective role in the development of colon carcinoma. Am J Cancer Res 5: 498-513.

76. Mellor KM, Varma U, Stapleton DI, Delbridge LM (2014) Cardiomyocyte glycophagy is regulated by insulin and exposure to high extracellular glucose. Am J Physiol Heart Circ Physiol 306: H1240-1245.

77. Reichelt ME, Mellor KM, Curl CL, Stapleton D, Delbridge LM (2013) Myocardial glycophagy - A specific glycogen handling response to metabolic stress is accentuated in the female heart. J Mol Cell Cardiol 65: 67-75.

78. Zhao Y, Zhang L, Qiao Y, Zhou X, Wu G, et al. (2013) Heme OxygenasePrevents Cardiac Dysfunction in Streptozotocin-Diabetic Mice by Reducing Inflammation, Oxidative Stress, Apoptosis and Enhancing Autophagy. PLoS One 8: e75927.

79. He W, Wang Q, Xu J, Xu X, Padilla M T, et al. (2012) Attenuation of TNFSF10 TRAIL-induced apoptosis by an autophagic survival pathway involving TRAF2and RIPK1/RIP1-mediated MAPK8/JNK activation. Autophagy 8: 1811-1821.

80. Senyo SE, Steinhauser ML, Pizzimenti CL, Yang VK, Cai L, et al. (2013) Mammalian Heart Renewal by Pre-existing Cardiomyocytes. Nature 493: 433436

81. Liu X, Liu C, Zhang X, Zhao J, Xu J (2015) Urocortin ameliorates diabetic cardiomyopathy in rats via the Akt/GSK-3 $\beta$ signaling pathway. Exp Ther Med 9: 667-674.

82. Huang Y, Yao X Q, Lau C W, Chan Y C, Tsang S Y, et al. (2004) Urocortin and cardiovascular protection. Acta Pharmacol Sin 25: 257-265

83. Li J, Qi D, Cheng H, Hu X, Miller E J, et al. (2013) Urocortin 2 autocrine/ paracrine and pharmacologic effects to activate AMP-activated protein kinase in the heart. Proc Natl Acad Sci U S A 110: 16133-16138.

84. Widyantoro B, Emoto N, Nakayama K, Anggrahini D W, Adiarto S, et al. (2010) Endothelial cell-derived endothelin-1 promotes cardiac fibrosis in diabetic hearts through stimulation of endothelial-to-mesenchymal transition. Circulation 121: 2407-2418.

85. Al-aryahi S, Kamato D, Getachew R, Zheng W, Potocnik S J, et al. (2014) Atherogenic, fibrotic and glucose utilising actions of glucokinase activators on vascular endothelium and smooth muscle. Cardiovasc Diabetol 13: 80.

86. Rother J, Richter C, Turco L, Knoch F, Mey I, et al. (2015) Crosstalk of cardiomyocytes and fibroblasts in co-cultures. Open Biol 5: 150038.

87. Bing R J, Hammond M M, Handelman J C, Powers R S, Spencer F, et al (1949) The measurement of coronary blood flow, oxygen consumption, and efficiency of the left ventricle in man. Am Heart J 38: 1-24.

88. Choi SW, Benzie IF, Ma SW, Strain JJ, Hannigan BM (2008) Acute hyperglycemia and oxidative stress: direct cause and effect?. Free Radic Biol Med 44: 1217-1231.

89. Brady M, Koban M U, Dellow K A, Yacoub M, Boheler K R, et al. (2003) Sp1 and Sp3 transcription factors are required for trans-activation of the human SERCA2 promoter in cardiomyocytes. Cardiovasc Res 60: 347-354.

90. Salameh A, Wustmann A, Karl S, Blanke K, Apel D, et al. (2010) Cyclic mechanical stretch induces cardiomyocyte orientation and polarization of the gap junction protein connexin43. Circ Res 106: 1592-1602.

91. Cagalinec M, Waczulíková I, Uličná O, Chorvat D Jr (2013) Morphology and contractility of cardiac myocytes in early stages of streptozotocin-induced diabetes mellitus in rats. Physiol Res 62: 489-501.

92. Jensen TE, Sylow L, Rose AJ, Madsen AB, Angin Y, et al. (2014) Contraction- stimulated glucose transport in muscle is controlled by AMPK and mechanical stress but not sarcoplasmatic reticulum $\mathrm{Ca}(2+)$ release. Mol Metab 3: 742-753.

93. Mehrotra A, Joe B, de la Serna IL L I (2014) Enhanced SWI/SNF enzyme recruitment and chromatin remodeling on fetal cardiac gene promoters is associated with cardiac hypertrophy in a genetic rat model of hypertension. Cell Physiol 228: 2337-2342.

94. Shimano M, Ouchi N, Walsh K (2012) Cardiokines: Recent Progress in Elucidating the Cardiac Secretome. Circulation 126: e327-332.

95. Hayakawa S, Ohashi K, Shibata R, Kataoka Y, Miyabe M, et al. (2015) Cardiac Myocyte-Derived Follistatin-Like 1 Prevents Renal Injury in a Subtotal Nephrectomy Model. J Am Soc Nephrol 26: 636-646.

96. Santopinto JJ, Fox KA, Goldberg RJ, Budaj A, Piñero G, et al. (2003) Creatinine clearance and adverse hospital outcomes in patients with acute coronary syndromes: findings from the global registry of acute coronary events (GRACE). Heart 89: 1003-1008.

97. Pitsavos C, Kourlaba G, Panagiotakos DB, Kogias Y, Mantas Y, et al. (2007) Association of Creatinine Clearance and In-Hospital Mortality in Patients With acute coronary syndromes: the GREECS study. Circ J 71: 9-14

98. Widera C, Giannitsis E, Kempf T, Korf-Klingebiel M, Fiedler B, et al. (2012) Identification of Follistatin-Like 1 by Expression Cloning as an Activator of the Growth Differentiation Factor 15 Gene and a Prognostic Biomarker in Acute Coronary Syndrome. Clin Chem 58: 1233-1241.

99. Kahli A, Guenancia C, Zeller M, Grosjean S, Stamboul K, et al. (2014) Growth Differentiation Factor-15 ( GDF-15 ) Levels Are Associated with Cardiac and Renal Injury in Patients Undergoing Coronary Artery Bypass Grafting with Cardiopulmonary Bypass. PLoS One 9: e105759.

100. Xu J, Kimball TR, Lorenz JN, Brown DA, Bauskin AR, et al. (2006) GDF15 / MIC-1 Functions As a Protective and Antihypertrophic Facto Released From the Myocardium in Association With SMAD Protein Activation. Circ Res 98: 342-350.

101. Russell NE, Higgins MF, Amaruso M, Foley M, McAuliffe FM (2009) Troponin $T$ and Pro - B-Type Natriuretic Peptide in Fetuses of Type 1 Diabetic. Diabetes Care 32: 2050-2055.

102. Maraj S, Patel R, Oliveros R, Sanon S, Dao T, et al. (2012) Potential Cardiometabolic Benefits of Renal Artery Denervation in Diabetics. J diabetes Metab S: 1-7.

103. Brandt MC, Mahfoud F, Reda S, Schirmer SH, Erdmann E, et al. (2012) Rena Sympathetic Denervation Reduces Left Ventricular Hypertrophy and Improves Cardiac Function in Patients With Resistant Hypertension. J Am Coll Cardio 59: 901-909.

104. Sharma R, Gaze D C, Pellerin D, Mehta R L, Gregson H, et al. (2006) Cardiac structural and functional abnormalities in end stage renal disease patients with elevated cardiac troponin T. Heart 92: 804-809.

105. Mahmoud SA, Poizat C (2013) Epigenetics and Chromatin Remodeling in Adult Cardiomyopathy. J Pathol 231: 147-157.

106. Mazzio EA, Soliman KF (2012) Basic concepts of epigenetics impact of environmental signals in gene expression. Epigenetics 7: 119-130.

107. Gaikwad AB, Sayyed SG, Lichtnekert J, Tikoo K, Anders H J (2010) Renal Failure Increases Cardiac Histone H3 Acetylation, Dimethylation, and Phosphorylation and the Induction of Cardiomyopathy-Related Genes in Type 2 Diabetes. Am J Pathol 176: 1079-1083.

108. Awad S, Al-Haffar KM, Marashly Q, Quijada P, Kunhi M, et al. (2015) Control of histone $\mathrm{H} 3$ phosphorylation by CaMKII in response to haemodynamic cardiac stress. J Pathol 235: 606-618.

109. Kadakol A, Malek V, Goru SK, Pandey A, Gaikwad AB (2015) Esculetin reverses histone $\mathrm{H} 2 \mathrm{~A}$ / $\mathrm{H} 2 \mathrm{~B}$ ubiquitination, $\mathrm{H} 3$ dimethylation, acetylation and phosphorylation in preventing type 2 diabetic cardiomyopathy. J Funct Foods 17: $127-136$.

110. Hang CT, Yang J, Han P, Cheng HL, Shang C, et al. (2010) Chromatin regulation by Brg1 underlies heart muscle development and disease. Nature 466: $62-67$.

111. Yang J, Xu W W, Hu SJ (2015) Heart Failure: Advanced Development in Genetics and Epigenetics. Biomed Res Int 2015: 352734

112. Xu J, Lei S, Liu Y, Gao X, Irwin M G, et al. (2013) Antioxidant N-Acetylcysteine 
Citation: Muralidaran Y, Viswanathan P (2015) Diabetic Cardiomyopathy: A New Perspective of Mechanistic Approach. J Diabetes Metab 6: 605. doi:10.4172/2155-6156.1000605

Attenuates the Reduction of Brg1 Protein Expression in the Myocardium of Type 1 Diabetic Rats. J Diabetes Res 2013: 716219.

113. Li H, Yao W, Irwin MG, Wang T, Wang S, et al. (2015) Adiponectin ameliorates hyperglycemia-induced cardiac hypertrophy and dysfunction by concomitantly activating Nrf2 and Brg1. Free Radic Biol Med 84: 311-21.

114. Liu ZZ, Zhao XZ, Zhang XS, Zhang M (2014) Promoter DNA demethylation of Keap1 gene in diabetic cardiomyopathy. Int J Clin Exp Pathol 7: 8756-8762.

115. Vecellio M, Spallotta F, Nanni S, Colussi C, Cencioni C, etal. (2014) The Histone Acetylase Activator Pentadecylidenemalonate 1b Rescues Proliferation and Differentiation in the Human Cardiac Mesenchymal Cells of Type 2 Diabetic Patients. Diabetes 63: 2132-2147.

116. Rausch JR (2010) Measures of Glycemic Variability and Links with Psychological Functioning. Curr Diab Rep 10: 415-421.

117. Nathan DM, Cleary PA, Backlund JY, Genuth SM, Lachin JM, et al. (2005) Intensive Diabetes Treatment and Cardiovascular Disease in Patients with Type 1 Diabetes The Diabetes Control and Complications Trial/Epidemiology of Diabetes Interventions and Complications (DCCT/EDIC) Study Research Group N Engl J Med 353: 2643-2653

118. Cushman WC, Evans GW, Byington RP, Goff DC Jr, Grimm RH Jr, etal. (2010) Effects of Intensive Blood-Pressure Control in Type 2 Diabetes Mellitus. N Engl J Med 362: 1575-1585.

119. Su G, Mi S, Tao H, Li Z, Yang H, et al. (2011) Association of glycemic variability and the presence and severity of coronary artery disease in patients with type 2 diabetes. Cardiovasc Diabetol 10: 19.

120.Boussageon R, Bejan-Angoulvant T, Saadatian-Elahi M, Lafont S Bergeonneau C, et al. (2011) Effect of intensive glucose lowering treatment on all cause mortality, cardiovascular death, and microvascular events in type 2 diabetes: meta-analysis of randomised controlled trials. BMJ 343: d4169.

121.Ceriello A (2012) The emerging challenge in diabetes: The "metabolic memory". Vascul Pharmacol 57: 133-138.

122. Ceriello A, Quagliaro L, Catone B, Pascon R, Piazzola M, et al. (2002) Role of hyperglycemia in nitrotyrosine postprandial generation. Diabetes Care 25 : 1439-1443.

123. Teno S, Uto $\mathrm{Y}$, Nagashima H, Endoh $\mathrm{Y}$, Iwamoto $\mathrm{Y}$, et al. (2000) Association of postprandial hypertriglyceridemia and carotid intima-media thickness in patients with type 2 diabetes. Diabetes Care 23: 1401-1406.

124. Ahmad J, Hameed B, Das G, Siddiqui MA, Ahmad I (2005) Postprandia hypertriglyceridemia and carotid intima-media thickness in north Indian type 2 diabetic subjects. Diabetes Res Clin Pract 69: 142-150.

125. Ceriello A, Esposito K, Piconi L, Ihnat MA, Thorpe JE, et al. (2008) Oscillating Glucose Is More Deleterious to Endothelial Function and Oxidative Stress Than Mean Glucose in Normal and Type 2 Diabetic Patients. Diabetes 57 1349-1354.

126. Piconi L, Quagliaro L, Da Ros R, Assaloni R, Giugliano D, et al. (2004) Intermittent high glucose enhances ICAM-1, VCAM-1, E-selectin and interleukin-6 expression in human umbilical endothelial cells in culture: the role of poly(ADP-ribose) polymerase. J Thromb Haemostv 2: 1453-1459.

127. Quagliaro L, Piconi L, Assaloni R, Da Ros R, Maier A, et al. (2005) Intermittent high glucose enhances ICAM-1, VCAM-1 and E-selectin expression in human umbilical vein endothelial cells in culture: the distinct role of protein kinase C and mitochondrial superoxide production. Atherosclerosis 183: 259-267.

128. Mellbin LG, Malmberg K, Rydén L, Wedel H, Vestberg D, et al. (2013) The relationship between glycaemic variability and cardiovascular complications in patients with acute myocardial infarction and type 2 diabetes: A report from the DIGAMI 2 trial. Eur Heart J 34: 374-379.

129. Scicchitano P, Cameli M, Maiello M, Modesti PA, Muiesan ML, et al. (2014) Nutraceuticals and dyslipidaemia: Beyond the common therapeutics. J Funct Foods 6: 11-32.

130. Ciccone MM, Scicchitano P, Cameli M, Cecere A, Cortese F, et al. (2014) Endothelial Function in Pre-diabetes, Diabetes and Diabetic Cardiomyopathy: A Review. J Diabetes Metab 5: 4.

131. Ciccone MM, Scicchitano P, Gesualdo M, Zito A, Carbonara S, et al. (2013) The role of omega- 3 polyunsaturated fatty acids supplementation in childhood: a review. Recent Pat Cardiovasc Drug Discov 8: 42-55.

132. Pannacciulli N, De Pergola G, Ciccone M, Rizzon P, Giorgino F, et al. (2003) Effect of family history of type 2 diabetes on the intima-media thickness of the common carotid artery in normal-weight, overweight, and obese glucosetolerant young adults. Diabetes Care 26: 1230-1234. 